\title{
Distributed Implementation of the Centralized Generalized Labeled Multi-Bernoulli Filter
}

\author{
Martin Herrmann, Student Member, IEEE, Charlotte Hermann, Michael Buchholz
}

\begin{abstract}
Distributed scenarios pose a big challenge to tracking and fusion systems. They require the prevention of repeatedly incorporating the same information, which originates from ring closures in the communication path and would affect optimality. Additionally, the multi-sensor multi-object Generalized Labeled Multi-Bernoulli filter update is NP-hard in principle. The method proposed in this paper tackles these problems, as it constitutes a divide and conquer strategy for distributed, synchronized multi-sensor systems with central fusion. Based on a common prediction, local sensor updates are calculated separately, sent back and fused centrally in order to start a new cycle. Thus, the intractable multi-sensor update is split into less complex local single-sensor updates and a novel, low-complexity fusion strategy. The proposed method enables a full parallelization of the optimal multi-sensor Generalized Labeled Multi-Bernoulli and $\delta$-Generalized Labeled Multi-Bernoulli update. Our approach bases on the Bayes Parallel Combination Rule and can be seen as multi-sensor multi-object Information Matrix Fusion for synchronous sensors, which constitutes a perfect choice in centralized systems with distributed sensors. Finally, we compare the proposed method to the Iterator Corrector approach from literature in detailed simulations.
\end{abstract}

Index Terms - State estimation, filtering, labeled random finite sets, multi-sensor multi-object tracking.

\section{INTRODUCTION}

$\mathbf{M}$ ULTI-sensor multi-object tracking aims to estimate the number of dynamic objects and their respective trajectories based on measurements of multiple sensors [1], [2]. While single-sensor systems are sometimes sufficient, many scenarios require the use of multiple sensors, e.g. due to sensor limitations, large environments or occlusions. One example is the field of Cooperative Intelligent Transportation Systems, where environment models from distributed infrastructure sensors [3], [4] can help automated vehicles [5] and human drivers [6], especially in complex urban scenarios [7].

For such scenarios, the tracking may be performed in a centralized or distributed manner. In the former, measurements (or measurement-equivalent data on a feature-level [8]) are passed to a centralized tracker, whereas in the latter, distributed trackers perform local estimations, which are then collected and fused wherever needed [2].

Manuscript received December 01, 2020, revised June 11, 2021 and August 09, 2021. Part of this work has been conducted as part of ICT4CART project which has received funding from the European Union's Horizon 2020 research \& innovation program under grant agreement No. 768953. Content reflects only the authors' view and European Commission is not responsible for any use that may be made of the information it contains.

M. Herrmann, C. Hermann and M. Buchholz are with the Institute of Measurement, Control and Microtechnology, Ulm University, Germany email: 〈firstname $\rangle.\langle$ lastname $\rangle @$ uni-ulm.de
Besides of Joint Probabilistic Data Association (JPDA) and Multi-Hypothesis Tracking (MHT) [1], the use of the Random Finite Set (RFS) framework [2] has been established in the field of multi-sensor multi-object tracking. This is notably due to the publication of the analytically solvable and first and only provably Bayes optimal multi-object filter, the Generalized Labeled Multi-Bernoulli (GLMB) filter [9] and its real-time capable implementation using equivalent $\delta$-Generalized Labeled Multi-Bernoulli ( $\delta$-GLMB) densities [10]. Other approaches like the Probability Hypothesis Density (PHD) filter [11], the Cardinalized Probability Hypothesis Density (CPHD) [12] filter, the Marginalized $\delta$-Generalized Labeled Multi-Bernoulli (M $\delta$-GLMB) [13] filter, or the Labeled Multi-Bernoulli (LMB) [14] filter are popular due to their low computational complexity. However, they involve (multiple) approximation stages, which have more or less significant impact on the estimation result [10], especially in complex multi-object scenarios. Furthermore, there are popular unlabeled approaches as the Multi-Target Multi-Bernoulli (MeMBer) filter [2] or the Cardinality Balanced Multi-Target Multi-Bernoulli (CB-MeMBer) filter [15], which suffer from the fact that no real trajectories are estimated, but only state estimations [16].

Concentrating on GLMB RFS, an often-used strategy in centralized and synchronous scenarios with multiple local sensors is the Iterator Corrector (IC). This was shown to be optimal, but intractable due to the involved NP-hard association problems [17]. In practice the density is truncated after each single-sensor update, which guarantees a manageable maximum complexity. However, the order of the single-sensor updates becomes relevant. In [18], a combined update of multiple sensor measurements is proposed. But it is also order dependent and infeasible in large sensor networks due to its quadratic complexity in the number of sensors and measurements. Finally, [17] proposes a very fast samplingbased update strategy, which jointly calculates the multisensor update for synchronous measurements. The approach is well suited for multi-sensor scenarios and outperforms the Iterator Corrector $\delta$-Generalized Labeled Multi-Bernoulli (IC- $\delta$-GLMB) filter in terms of precision, while having similar computational complexity [17]. However, both approaches require conditionally independent sensor measurements, which is often assumed true, especially using the standard multiobject measurement model [2].

Considering fully distributed scenarios with arbitrary network topology, where information is shared between multiple agents directly and in parallel, double counting of shared 
information must be prohibited [1]. Calculation and removal of this information prior to the fusion at each node is possible, but computationally complex and, therefore, often infeasible in practical systems. Thus, only sub-optimal track-to-track fusion schemes, which effectively suppress the incorporation of common information on the cost of a sub-optimal information gain, are available. These methods can roughly be categorized into one of two principled strategies that rely on the idea of either linear opinion pooling or logarithmic opinion pooling [19]. Then, methods of the former kind are often referred to as Arithmetic Mixture Density (AMD) [20] or Arithmetic Average (AA) [21], and methods of the latter kind are often denoted Generalized Covariance Intersection (GCI) [22], [23], Geometric Mixture Density (GMD) [24], Kullback-Leibler Average (KLA) [25], Exponential Mixture Density (EMD) or Geometric Average (GA) [21].

Recent developments applying track-to-track fusion methods to the RFS framework and open challenges have been summarized in the survey papers [24], [26]. The GCI has become very popular and has been applied to various kinds of RFSs, e.g. [27], [28], [29], [30], [31], [32]. Although it has been reported that GCI tends to be less prone to clutter, it has problems with misdetections, object birth, local pruning, and non-overlapping sensor Field of Views (FOVs) [21], [26]. As discussed in [31], this results in label mismatches and decreased fusion performance, which can be measured by a label inconsistency indicator [33]. Further investigations on LMB densities with a soft-decision strategy to minimize the label inconsistency indicator showed a performance close to that of an Iterator Corrector Labeled Multi-Bernoulli (IC-LMB) filter in specific scenarios [34]. Newer literature [21], [26], however, indicates that the AMD fusion outperforms the GCI method in terms of computational efficiency as well as performance. Thus, there is an increasing interest on the AMD fusion rule, e.g. [21], [35], [36], [37], [38].

All these methods constitute promising approaches, but are intended to be used in scenarios that lack from the general problem of unknown statistical dependencies between local sensor posterior densities. Thus, none of these methods yields a Bayes optimal solution and suits centralized scenarios, where this information is available. Consequently, the application of track-to-track approaches is limited to fully distributed scenarios, which rise a totally different challenge than centralized ones.

But even in rather organized scenarios with centralized fusion and distributed sensors, further limitations, like the requirement to transmit raw measurements or feature-level measurements [8], are present. While the transmission of raw measurements is often infeasible, e.g. for high-definition image data in wireless networks, the use of feature-level measurements may discard useful information and limits the measurement model. The inverse lidar measurement model of [39], for example, relies on no-target information and cannot be reasonably applied to a system using feature-level measurements. Even without such limitations, the centralized tracker must implement the measurement model. Hence, in practical systems, where components of different suppliers are used together, intellectual property may be concerned and complex interfaces are required, like our ultra-flexible and generic feature-level measurement model [40].

Yet another approach for such scenarios is the Information Matrix Fusion (IMF), which, however, is only available for the single-object case. It originates from the Bayes Parallel Combination Rule (BPCR) [41], [2] and relies on the rather typical assumption of independent sensor measurements. Then, the IMF allows a parallel update for all sensor measurements and yields a closed-form solution for the fusion at the central fusion node. This strategy is known to be optimal for the Kalman filter and is denoted distributed implementation of the centralized estimator with central fusion [1]. Similarly, there exist a few multi-object implementations for RFS densities based on the BPCR [42], [43], but none of them is Bayesoptimal or uses GLMB RFSs. The use of such BPCR based filters, though, has been identified potentially reasonable, and finding a computationally efficient and reliable solution has explicitly be mentioned as an open research problem in [26].

Motivated by this analysis revealing the lack of a Bayesoptimal filter for distributed multi-sensor multi-object tracking including a tractable implementation, this paper presents a novel distributed implementation of the centralized multisensor multi-object estimator with central fusion and synchronous sensors. In particular,

1) We propose the Product Multi-Sensor Generalized Labeled Multi-Bernoulli (PM-GLMB) filter, which constitutes a Bayes-optimal solution, as it is the first implementation of the BPCR for GLMB densities. The filter is described in Section III and allows to shift the computation of the update to the distributed sensor nodes, and thus yields a full parallel update. With that, our method also meets the demand for intellectual property protection of sensor manufacturers.

2) Based on this, we present a computationally efficient and optimal fusion strategy that allows truncation of insignificant hypotheses prior to their calculation in Section IV.

3) In Section V, we compare our approach against the quasi-standard IC- $\delta$-GLMB filter and show that it yields a substantial performance improvement that goes along with an increasing number of sensors. These results further show that our filter is beneficial even in purely centralized systems.

Before we present and evaluate our proposed method, Section II summarizes the notation and background on Bayes estimation required for our derivations. The paper closes with some conclusion and an outlook on future work in Section VI.

\section{BACKGROUND AND NOTATION}

In the first part, this section focuses on IMF as a distributed implementation of the multi-sensor single-object Bayes filter. This is followed by a summary of the multi-sensor multiobject Bayes filter based on the Finite Set Statistics (FISST) framework [2]. Furthermore, the GLMB and $\delta$-GLMB filter update formulas are given as a basis for our proposed filter.

\section{A. Notation}

We inherit our notation from [17], which is summarized in what follows. Single objects are represented by a vector- 
wise random variable drawn from an underlying space and denoted by small letters, like the object's state $x \in \mathbb{X}$ in the state space $\mathbb{X}$ and a single-object measurement $z \in \mathbb{Z}$ in the the measurement space $\mathbb{Z}$. Multiple objects are represented by random variables defined on the hyperspace of all finite subsets $\mathcal{F}(\cdot)$ of the underlying space. They are modeled using RFSs [2], denoted by capital letters, like the multi-object state RFS $X$ on $\mathcal{F}(\mathbb{X})$ and the multi-object measurement RFS $Z$ on $\mathcal{F}(\mathbb{Z})$. To distinguish RFSs from matrices, which are also denoted in capital letters, the latter are additionally underlined. Further, the operator $(\cdot)_{m: n, j: k}$ refers to a submatrix or element composed of the $m$-th to the $n$-th row and the $j$-th to the $k$-th column of a matrix or vector.

The statistical properties of single-object states are described using probability density functions denoted by $p$. The statistics of multi-object states are described using the multiobject density function counterpart [2] denoted by $\pi$. Both are referred to as density within this article, since a distinction is always easily possible. Statistical independence is denoted by $\perp$, e.g. $p_{A} \perp p_{B}$, and $\left(p_{A} \perp p_{B}\right) \mid p_{C}$ denotes conditional independence of $p_{A}$ and $p_{B}$ given $p_{C}$.

Labeled RFSs are constructed by augmenting the states by a label $\ell \in \mathbb{L}$, i.e. $\boldsymbol{x}=(x, \ell)$. In order to distinguish between labeled and unlabeled densities and states, the labeled versions are denoted by bold symbols. Thus, $\boldsymbol{X}$ is a labeled RFS on the labeled state space $\mathbb{X} \times \mathbb{L}$. The set of labels of an RFS is extracted using the projection function $\mathcal{L}: \mathbb{X} \times \mathbb{L} \rightarrow \mathbb{L}$ defined by $\mathcal{L}(\boldsymbol{x})=\ell$, thus, $\mathcal{L}(\boldsymbol{X})=\{\mathcal{L}(\boldsymbol{x}): \boldsymbol{x} \in \boldsymbol{X}\}$.

The inner product is abbreviated by $\langle f, g\rangle \triangleq \int f(x) g(x) d x$. Further, the multi-object exponential is defined by $h^{X} \triangleq$ $\prod_{x \in X} h(x)$, where $h^{X}=1$ if $X=\emptyset$, and the generalized Kronecker delta function is given by

$$
\delta_{Y}(X) \triangleq\left\{\begin{array}{ll}
1 & , \text { if } X=Y \\
0 & , \text { otherwise. }
\end{array} .\right.
$$

Moreover, association hypotheses connecting track labels and measurements are denoted by $\theta: \mathbb{L} \rightarrow 0:|Z|$, where " 0 " represents the missed detection and $\theta^{-1}$ denotes the inverse mapping. Here, the set of all mappings is denoted $\Theta$ and $|Z|$ denotes the number of elements in the measurement RFS. Finally, the distinct label indicator $\Delta(\boldsymbol{X})=\delta_{|\boldsymbol{X}|}(|\mathcal{L}(\boldsymbol{X})|)$ is used to exclude invalid realizations of an RFS.

Within all multi-sensor equations, time indices are omitted to simplify notation, wherever the context allows easy distinction. Predicted densities are, however, marked by a '+' subscript. Moreover, the following multi-sensor abbreviations are used:

$$
\begin{gathered}
z \triangleq\left(z^{(1)}, \ldots, z^{(V)}\right), \quad Z \triangleq\left(Z^{(1)}, \ldots, Z^{(V)}\right), \\
\Theta \triangleq \Theta^{(1)} \times \ldots \times \Theta^{(V)}, \quad \theta \triangleq\left(\theta^{(1)}, \ldots, \theta^{(V)}\right), \\
\delta_{\theta^{-1}(\{0:|Z|\})}(L) \triangleq \prod_{s=1}^{V} \delta_{\theta^{(s)-1}\left(\left\{0:\left|Z^{(s)}\right|\right\}\right)}(L), \\
\psi_{Z}(x, \ell, \theta) \triangleq \prod_{s=1}^{V} \psi_{Z^{(s)}}\left(x, \ell, \theta^{(s)}\right),
\end{gathered}
$$

where $V$ is the number of involved sensors and $s \in\{1, \ldots, V\}$ denotes the actual sensor. To distinguish between a global multi-sensor symbol and its local counterpart, the superscript ' $(s)$ ' is added to the local symbol, e.g. $z$ denotes a multi-sensor measurement and $z^{(s)}$ the s-th sensor's measurement.

\section{B. Bayes Estimation}

The goal of multi-sensor object tracking is to calculate a probabilistic estimate about the number of objects under observation and their state based on prior knowledge, measurements and background information. To make this task feasible, the Markov assumption is applied, i.e. an object's state subsumes the information from all prior states. With that, the Bayes filter formulas describe a feasible strategy to calculate the wanted density iteratively in a prediction and an update step [2]. In the single-object case, the update is defined by

$$
p_{k \mid k}\left(x \mid z_{1: k}\right)=p_{k \mid k-1}\left(x \mid z_{1: k-1}\right) \cdot \frac{g_{k}\left(z_{k} \mid x\right)}{p_{k}\left(z_{k} \mid z_{1: k-1}\right)},
$$

where $z_{1: k-1}$ is the time series of the sensor measurements, $x$ is the true state of the object under observation, and $g_{k}\left(z_{k} \mid x\right)$ is the measurement likelihood density, all at time instance $k$.

Omitting the time indices and switching to the simplified notation introduced in Section II-A, the update formula for the multi-object case is given by

$$
\boldsymbol{\pi}(\boldsymbol{X} \mid Z)=\boldsymbol{\pi}_{+}(\boldsymbol{X}) \cdot \frac{g(Z \mid \boldsymbol{X})}{\pi(Z)},
$$

where the abbreviation $\pi(Z) \triangleq \pi\left(Z_{k} \mid Z_{1: k-1}\right)$ is used. Similar to before, $\boldsymbol{X}$ denotes the true labeled multi-object state, $Z$ is the multi-object measurement set, $g(Z \mid \boldsymbol{X})$ is the multi-object measurement likelihood density, and $\boldsymbol{\pi}_{+}(\boldsymbol{X})$ is the predicted multi-object density.

\section{Multi-Sensor Single-Object Kalman Filter}

The following section is based on [1] and summarizes the Kalman filter as well as the IMF equivalent briefly.

The Kalman filter formulas yield a closed form solution to the Bayes filter update, if the initial state estimate is Gaussian distributed with mean vector $\hat{x}_{k}$ and covariance matrix $\underline{P}_{k}$ and if linear Gaussian measurement models are applied. Such measurements are modeled by $z_{k}=\underline{H}_{k} x+n_{k}$, where $x$ is the true state of the object under observation. Further, $n$ is a multi-variate zero-mean Gaussian distributed random variable with covariance matrix $\underline{R}_{k}$, which models the measurement noise, and $\underline{H}_{k}$ is the measurement matrix, which maps from the state space to the measurement space. Since the Gaussian distribution has been shown to be conjugate with respect to a Gaussian likelihood function, the calculation of the posterior distribution reduces to the calculation of the mean and covariance. The filter update formulas with filter gain $\underline{K}_{k}$ and innovation covariance $\underline{S}_{k}$ are given by

$$
\begin{aligned}
\underline{S}_{k} & =\underline{H}_{k} \underline{P}_{k \mid k-1} \underline{H}_{k}^{T}+\underline{R}_{k}, \\
\underline{K}_{k} & =\underline{P}_{k \mid k-1} \underline{H}_{k}^{T} \underline{S}_{k}^{-1}, \\
\hat{x}_{k} & =\hat{x}_{k \mid k-1}+\underline{K}_{k}\left(z_{k}-\underline{H}_{k} \hat{x}_{k \mid k-1}\right), \\
P_{k} & =\underline{P}_{k \mid k-1}-\underline{K}_{k} \underline{S}_{k} \underline{K}_{k}^{T} .
\end{aligned}
$$


Let us assume $V$ synchronous sensors with mutually independent measurements, i.e. $z_{k}^{(i)} \perp z_{k}^{(j)}, \forall i \neq j$, and conditionally independent measurements given the true state, i.e. $\left(z_{k}^{(i)} \perp z_{k}^{(j)}\right) \mid x, \forall i \neq j$. Then, the multi-sensor measurement likelihood density is given by

$$
g_{k}\left(z_{k} \mid x\right)=\prod_{s=1}^{V} g_{k}^{(s)}\left(z_{k}^{(s)} \mid x\right) .
$$

Both independence conditions hold for the linear Gaussian measurement model. Thus, the Kalman filter formulas (5) can iteratively be applied, which is called the IC, providing a simple solution to the multi-sensor single-object case.

From the independence, it follows that the multi-sensor measurement function $\underline{H}_{k}$ is created by vertically stacking the single-sensor measurement functions $\underline{H}_{k}^{(s)}$. The multi-sensor measurement uncertainty matrix $\underline{R}_{k}=\operatorname{diag}\left(\left[\underline{R}_{k}^{(1)} \cdots \underline{R}_{k}^{(V)}\right]\right)$ has block diagonal form, which can be reused to obtain the IMF formulas:

$$
\begin{aligned}
\underline{P}_{k}^{-1} & =\underline{P}_{k \mid k-1}^{-1}+\sum_{s=1}^{V}\left(\underline{H}_{k}^{(s)}\right)^{T}\left(\underline{R}_{k}^{(s)}\right)^{-1} \underline{H}_{k}^{(s)} \\
& =\underline{P}_{k \mid k-1}^{-1}+\sum_{s=1}^{V} \underline{I}_{k}^{(s)}, \\
\underline{P}_{k}^{-1} \hat{x}_{k} & =\underline{P}_{k \mid k-1}^{-1} \hat{x}_{k \mid k-1}+\sum_{s=1}^{V}\left(\underline{H}_{k}^{(s)}\right)^{T}\left(\underline{R}_{k}^{(s)}\right)^{-1} z_{k}^{(s)} \\
& =\underline{P}_{k \mid k-1}^{-1} \hat{x}_{k \mid k-1}+\sum_{s=1}^{V} i_{k}^{(s)} .
\end{aligned}
$$

This allows to separate an independent information part consisting of the information matrices $\underline{I}_{k}^{(s)}$ and information vectors $i_{k}^{(s)}$ from the local posterior densities. Thus, the sensor updates can be separated from each other, which makes the filter perfectly suitable for scenarios with centralized fusion node and distributed sensors. In such a configuration, the sensors transmit the information part, which is simply summed up in the central tracker to gain the global updated density.

\section{Multi-Object Generalized Labeled Multi-Bernoulli Filter}

The GLMB density has been shown to be conjugate with respect to the standard multi-object measurement likelihood density and is closed under the Chapman-Kolmogorov equation [9]. Furthermore, the GLMB filter is Bayes optimal [43] and a tractable implementation using equivalent $\delta$-GLMB densities is available. Both are introduced briefly in the following.

1) Generalized Labeled Multi-Bernoulli: An RFS is denoted GLMB [9] if its multi-object density is defined by

$$
\boldsymbol{\pi}(\boldsymbol{X})=\Delta(\boldsymbol{X}) \sum_{c \in \mathbb{C}} w^{(c)}(\mathcal{L}(\boldsymbol{X}))\left[p^{(c)}\right]^{\boldsymbol{X}}
$$

Here, $c \in \mathbb{C}$ drawn from a discrete index set enumerates the GLMB hypotheses composed of the product of single-object densities $p^{(c)}(\boldsymbol{x})$ and label-dependent weight $w^{(c)}(\mathcal{L}(\boldsymbol{X}))$.
2) $\delta$-Generalized Labeled Multi-Bernoulli: $\delta$-GLMB RFS [9] constitute a special, but in general equivalent RFS to the GLMB. Their density is defined by

$$
\boldsymbol{\pi}(\boldsymbol{X})=\Delta(\boldsymbol{X}) \sum_{(I, \xi) \in \mathcal{F}(\mathbb{L}) \times \Xi} w^{(I, \xi)} \delta_{I}(\mathcal{L}(\boldsymbol{X}))\left[p^{(\xi)}\right]^{\boldsymbol{X}},
$$

where the following parameters of (8) have been substituted:

$$
\begin{aligned}
C & =\mathcal{F}(\mathbb{L}) \times \Xi, \\
w^{(c)}(L) & =w^{(I, \xi)} \delta_{I}(L), \\
p^{(c)} & =p^{(I, \xi)}=p^{(\xi)} .
\end{aligned}
$$

Since the spatial distribution of an object does not depend on the total label set in $\boldsymbol{X}$, but on its history of track label to measurement associations, there exist multiple copies of identical spatial distributions in a GLMB density. Hence, the underlying idea of these substitutions is to remove these duplicates to reduce the computational complexity of the filter. To do so, a hypothesis is identified by the so-called support $(I, \xi)$. Here, $I \in \mathcal{F}(\mathbb{L})$ is a label set drawn from all finite subsets of the label space $\mathbb{L}$ and $\xi \in \Xi$ is a history of track label to measurement association maps, drawn from all possible association histories $\Xi$, i.e. $\xi=\left(\theta_{1: k}\right)$. Then, the distinct label indicator $\delta_{I}(\mathcal{L}(\boldsymbol{X}))$ is required to reject inconsistent pairs $(I, \xi)$ by assignment of a zero weight, and (10c) follows.

3) Generalized Labeled Multi-Bernoulli Filter: The standard formulation of the multi-sensor GLMB filter [17] relies on the standard single-sensor multi-object measurement model [43], which considers misdetections as well as clutter events. Then, $Z^{(s)}=\left\{z_{1}^{(s)}, \ldots, z_{m}^{(s)}\right\}$ is a measurement set, where each object $i$ gives rise to at most one single-object measurement $z_{i}^{(s)}=\underline{H}^{(s)} x_{i}+n_{i}^{(s)}$ (see Section II-C), where $p_{D}^{(s)}(x, \ell)=$ $1-q_{D}^{(s)}(x, \ell)$ is the detection probability of the object with label $\ell$. Then, independence and conditional independence of multi-object measurements of different sensors hold, and the multi-sensor updated posterior density is given by

$$
\boldsymbol{\pi}(\boldsymbol{X} \mid Z)=\Delta(\boldsymbol{X}) \sum_{c \in \mathbb{C}} \sum_{\theta \in \Theta} w_{Z}^{(c, \theta)}(\mathcal{L}(\boldsymbol{X}))\left[p^{(c, \theta)}(\cdot \mid Z)\right]^{\boldsymbol{X}},
$$

if the prior density is a GLMB density with predicted weights $w_{+}^{(c)}(\mathcal{L}(\boldsymbol{X}))$ and predicted spatial distributions $p_{+}^{(c)}(x, \ell)$. Based on the number of track label to measurement association maps $|\Theta|$, each hypothesis generates a set of new hypotheses with the updated parameters given by

$$
w_{Z}^{(c, \theta)}(L)=C_{d} \delta_{\theta^{-1}(\{0:|Z|\})}(L) w_{+}^{(c)}(L)\left[\eta_{Z}^{(c, \theta)}(\cdot)\right]^{L},
$$

$$
\begin{aligned}
\eta_{Z}^{(c, \theta)}(\ell) & =\left\langle p_{+}^{(c)}(\cdot, \ell), \psi_{Z}(\cdot, \ell, \theta)\right\rangle, \\
p^{(c, \theta)}(x, \ell \mid Z) & =\frac{p_{+}^{(c)}(x, \ell) \psi_{Z}(x, \ell, \theta)}{\eta_{Z}^{(c, \theta)}(\ell)}, \\
\psi_{Z}(x, \ell, \theta) & =\prod_{s=1}^{V} \psi_{Z^{(s)}}\left(x, \ell, \theta^{(s)}\right),
\end{aligned}
$$




$$
\begin{aligned}
& \psi_{Z^{(s)}}\left(x, \ell ; \theta^{(s)}\right)=\delta_{0}\left(\theta^{(s)}(\ell)\right) q_{D}^{(s)}(x, \ell) \\
& \quad+\left(1-\delta_{0}\left(\theta^{(s)}(\ell)\right)\right) \frac{p_{D}^{(s)}(x, \ell) g^{(s)}\left(z_{\theta^{(s)}(\ell)} \mid x, \ell\right)}{\kappa^{(s)}\left(z_{\theta^{(s)}(\ell)}\right)} .
\end{aligned}
$$

Here, $w_{Z}^{(c, \theta)}(L)$ denotes the hypotheses weights, which depend on the actual label set $L$, and $p^{(c, \theta)}(x, \ell \mid Z)$ is the spatial distribution of object $x$ with label $\ell$, given the association $\theta$. They are normalized by the sum of all weights $C_{d}^{-1}$ and the likelihood of a specific track label to measurement association $\eta_{Z}^{(c, \theta)}(\ell)$, respectively. Furthermore, due to the independence assumption and the sensor synchronicity, the generalized multi-sensor multi-object measurement likelihood density $\psi_{Z}(x, \ell, \theta)$ is the product of the independent generalized multi-object measurement likelihood densities $\psi_{Z^{(s)}}\left(x, \ell ; \theta^{(s)}\right)$ of the sensors. Here, $\kappa^{(s)}\left(z_{\theta^{(s)}(\ell)}\right)$ is the intensity function of Poisson distributed clutter events and $g^{(s)}\left(z_{\theta^{(s)}(\ell)} \mid x, \ell\right)$ denotes the single-object measurement likelihood density for measurement $z_{\theta^{(s)}(\ell)}$ associated with track $\ell$ and state $x$.

Given the case that all predicted single-object densities are Gaussian distributions of the form $p_{+}^{(c)}(x, \ell)=\mathcal{N}\left(x ; \hat{x}_{+}^{(c, \ell)}, \underline{P}_{+}^{(c, \ell)}\right)$ and all measurement models are linear Gaussian, the mean and the covariance sufficiently describe the updated single-object density. It is given by

$$
p^{(c, \theta)}(x, \ell)=\mathcal{N}\left(x ; \hat{x}^{(c, \ell, \theta)}, \underline{P}^{(c, \ell, \theta)}\right),
$$

with $\hat{x}^{(c, \ell, \theta)}$ and $\underline{P}^{(c, \ell, \theta)}$ given similar to $(7 \mathrm{c})$ and $(7 \mathrm{a})$, respectively (see [10] for more details).

4) $\delta$-Generalized Labeled Multi-Bernoulli Filter: The updated posterior density of the $\delta$-GLMB filter is akin to (11) after application of the substitutions (10). Applying the same assumptions as for the GLMB filter, it is given by

$$
\begin{aligned}
& \boldsymbol{\pi}(\boldsymbol{X} \mid Z)=\Delta(\boldsymbol{X}) \sum_{(I, \xi) \in \mathcal{F}(\mathbb{L}) \times \Xi} \sum_{\theta \in \Theta} w^{(I, \xi, \theta)}(Z) \\
& \delta_{I}(\mathcal{L}(\boldsymbol{X}))\left[p^{(\xi, \theta)}(\cdot \mid Z)\right]^{\boldsymbol{X}} .
\end{aligned}
$$

The parameters follow straightforwardly from (12) with the substitutions (10).

\section{Multi-Sensor Tracking FOR Synchronous SENSORS}

Both, the GLMB as well as the $\delta$-GLMB filter allow for iterative application of the filter update step for synchronous measurements. This constitutes the Iterator Corrector Generalized Labeled Multi-Bernoulli (IC-GLMB) and IC- $\delta$-GLMB filter and is possible due to the product in (12d). In principal, this strategy yields the exact solution and is independent of the sensor ordering [17]. However, the order becomes relevant if the respective posterior densities are truncated or if approximations take place, which is required in practical systems due to the super-exponential growth in the number of hypotheses. For central updates, the sub-optimal Gibbs sampler based $\delta$-GLMB (SO-Gibbs- $\delta$-GLMB) filter [17] provides an approximative and computationally cheap all-in-one multi-sensor solution. However, a distributed calculation of the sensor updates is not possible with this approach. In contrast, our proposed filter constitutes a totally different solution for the sensor updates by introducing a divide and conquer strategy, while it still bases on a central fusion. According to the terminology of [9], [42], our approach is denoted PM-GLMB filter or, if its implementation is based on $\delta$-GLMB densities, Product MultiSensor $\delta$-Generalized Labeled Multi-Bernoulli (PM- $\delta$-GLMB) filter. Closed form solutions for both filters are derived in the end of this section. Beforehand, we summarize the underlying BPCR and add some remarks about the application of the Kalman filter assumptions to it, which yields a different derivation approach of the well-known IMF.

\section{A. Bayes Parallel Combination Rule}

The BPCR can be derived from the standard Bayes multisensor formula for synchronous sensors by subtle reformulation [2], [41]. It allows a very intuitive interpretation of the common information shared between all posteriors in distributed implementations of the centralized estimator with central fusion. This simply corresponds to the prior information, i.e. the central prediction. Hence, the common information is already known in such systems, which renders its complex computation unnecessary. But the approach requires the local densities to be divided by the predicted density $V-1$ times.

Application of the BPCR to the multi-sensor single-object and multi-object cases and $V$ synchronous sensors yields

$$
\begin{aligned}
p_{k \mid k}\left(x \mid z_{1: k}\right) & =C_{p} \frac{p_{k \mid k}^{(1)}\left(x \mid z_{1: k}^{(1)}\right) \cdot \ldots \cdot p_{k \mid k}^{(V)}\left(x \mid z_{1: k}^{(V)}\right)}{\left(p_{k \mid k-1}(x) \mid z_{1: k-1}\right)^{V-1}}, \\
\boldsymbol{\pi}(\boldsymbol{X} \mid Z) & =C_{p} \frac{\prod_{s=1}^{V} \boldsymbol{\pi}^{(s)}\left(\boldsymbol{X} \mid Z^{(s)}\right)}{\left(\boldsymbol{\pi}_{+}(\boldsymbol{X})\right)^{V-1}}
\end{aligned}
$$

respectively. In both equations, the normalization constant $C_{p}$ compensates for a substitution in the denominators when deriving them. Both expressions allow for distributed sensor updates, if the appropriate division of densities is possible, since all sensor updates can be calculated based on the central prediction and the measurements only. Further, both formulas account for the truncation of shared information in the case of synchronous sensor measurements. A generalization of this rule for arbitrary common information can be found in [44].

\section{B. Distributed Implementation of the Kalman Filter}

Based on the BPCR, a distributed implementation of the centralized IMF can be derived [44]. It is equivalent to the information filter form of the Kalman filter [1] and constitutes a special case, since no feedback channel is required. It is given by

$$
\begin{aligned}
p_{k \mid k}(x \mid z) & =C_{p} \frac{\prod_{s=1}^{V} \mathcal{N}\left(x ; \hat{x}_{k}^{(s)}, P_{k}^{(s)}\right)}{\left(\mathcal{N}\left(x ; \hat{x}_{k \mid k-1}, P_{k \mid k-1}\right)\right)^{V-1}} \\
& =\mathcal{N}\left(x ; \hat{x}_{k}, \underline{P}_{k}\right),
\end{aligned}
$$

where $\underline{P}_{k}$ and $\hat{x}_{k}$ can be derived from (7). 
The outline of the derivation allows a deeper insight on the abstract term of common information. First, the Gaussian product and division in (16a) is dissolved, which yields $p_{k \mid k}(x \mid z)=\mathcal{N}\left(x ; \hat{x}_{k}, \underline{P}_{k}\right)$, where

$$
\underline{P}_{k}^{-1}=\sum_{s=1}^{V}\left(\underline{P}_{k}^{(s)}\right)^{-1}-(V-1)\left(\underline{P}_{k \mid k-1}\right)^{-1} .
$$

Then, subtle reformulation using the matrix inversion lemma [45] and repetition of similar steps for the state $\hat{x}_{k}$ complete the proof. Equations (16a) and (17) show that the common information has to be removed after naive fusion of the sensor updates and that this common information equals the central prediction. This is evident from the $V-1$-fold appearance of the predicted density in the denominator of (16a), as well as the covariance in (17) as subtrahend. Thus, the IMF benefits from the fact that the common information can be separated and is already known due to the system design.

\section{Distributed Implementation of the Generalized Labeled Multi-Bernoulli Update}

As mentioned earlier, we denote our distributed implementation of the centralized GLMB fusion PM-GLMB filter, according to the conventions of [9], [42]. The PM-GLMB filter considers the statistical dependencies between the local sensor posterior densities by the $V-1$-fold division with the predicted density. Note that our approach renders this possible, although there is no general solution for a division by a GLMB density.

Proposition 3.1: Let us assume $V$ local posterior updated GLMB densities $\boldsymbol{\pi}^{(s)}\left(\boldsymbol{X} \mid Z^{(s)}\right)$, whose spatial distributions are Gaussian (cf. (13)). Furthermore, these local densities base on a common predicted GLMB density, $p_{D}^{(s)}(x, l)=p_{D}^{(s)}$, and all measurement models are linear Gaussian, i.e. go along with the independence criteria of Section II-C. Then, the fused updated posterior density $\boldsymbol{\pi}(\boldsymbol{X} \mid Z)$ is a GLMB multi-object distribution and yields

$$
\boldsymbol{\pi}(\boldsymbol{X} \mid Z)=\Delta(\boldsymbol{X}) \sum_{c \in \mathbb{C}} \sum_{\theta \in \Theta} \tilde{w}_{Z}^{(c, \theta)}(\mathcal{L}(\boldsymbol{X}))\left[\tilde{p}^{(c, \theta)}(\cdot \mid Z)\right]^{\boldsymbol{X}}
$$

where

$$
\begin{aligned}
\tilde{w}_{Z}^{(c, \theta)}(L) & =\frac{\tilde{C}_{w} \prod_{s=1}^{V} w_{Z\left({ }^{(s)}\right.}^{\left(c, \theta^{(s)}\right.}(L)}{\left[w_{+}^{(c)}(L)\right]^{V-1}}, \\
\tilde{p}^{(c, \theta)}(x, \ell \mid Z) & =\frac{\tilde{C}_{p}(x, \ell \mid Z) \prod_{s=1}^{V} p^{\left(c, \theta^{(s)}\right)}\left(x, \ell \mid Z^{(s)}\right)}{\left[p_{+}^{(c)}(x, \ell)\right]^{V-1}} \\
& =\mathcal{N}\left(x ; \hat{x}^{(c, \ell, \theta)}, \underline{P}^{(c, \ell, \theta)}\right) .
\end{aligned}
$$

The normalization constant $\tilde{C}_{p}(x, \ell \mid Z)$ compensates a constant from the product and the division of the densities (compare to Section III-B), and $\tilde{C}_{w}$ is a global constant ensuring correct normalization of the weights. Further, the spatial densities are calculated by applying the IMF formulas (7), yielding

$$
\begin{aligned}
& \underline{P}^{(c, \ell, \theta)}=\left(\left(\underline{P}_{+}^{(c, \ell)}\right)^{-1}+\sum_{s=1}^{V} \underline{I}^{\left(c, \ell, \theta^{(s)}\right)}\right)^{-1} \\
& \hat{x}^{(c, \ell, \theta)}=\underline{P}^{(c, \ell, \theta)}\left(\left(\underline{P}_{+}^{(c, \ell)}\right)^{-1} \hat{x}_{+}^{(c, \ell)}+\sum_{s=1}^{V} i^{\left(c, \ell, \theta^{(s)}\right)}\right),
\end{aligned}
$$

where $\underline{I}$ and $i$ denote the information matrix and information vector analogously defined to (7b) and (7d), respectively.

Proof: Since a GLMB density is fully characterized by its weights $w^{(c, \theta)}$ and spatial distributions $p^{(c, \theta)}$, it is sufficient to show the equality of the weights $((18 b)$ and (12a)) and the equality of the spatial densities ((18d) and (12c)).

First, we proof the equality of the weights. The product of the predicted density and the multi-sensor measurement likelihood yields

$$
\begin{aligned}
p_{+}^{(c)}(\cdot, \ell) \cdot \psi_{Z}(\cdot, \ell ; \theta) \\
=\mathcal{N}\left(x ; \hat{x}_{+}^{(c, \ell)}, \underline{P}_{+}^{(c, \ell)}\right) \prod_{s=1}^{V}\left(\delta_{0}\left(\theta^{(s)}(\ell)\right) q_{D}^{(c, \ell, s)}\right. \\
\left.\quad+\left(1-\delta_{0}\left(\theta^{(s)}(\ell)\right)\right) \alpha^{(c, \ell, s)} \mathcal{N}\left(z_{\theta^{(s)}(\ell)} ; \underline{H}^{(s)} x, \underline{R}^{(s)}\right)\right) \\
=\mathcal{N}\left(x ; \hat{x}^{(c, \ell, \theta)}, \underline{P}^{(c, \ell, \theta)}\right) \prod_{s=1}^{V}\left(\delta_{0}\left(\theta^{(s)}(\ell)\right) q_{D}^{(c, \ell, s)}\right. \\
\left.\quad+\left(1-\delta_{0}\left(\theta^{(s)}(\ell)\right)\right) \alpha^{(c, \ell, s)} \mathcal{N}\left(z_{\theta^{(s)}(\ell)} ; z_{+}^{(c, \ell, s)}, \underline{S}^{(c, \ell, s)}\right)\right) .
\end{aligned}
$$

Here, the fundamental Gaussian identity [46] has been applied, thus, $z_{+}^{(c, \ell, s)}=\underline{H}^{(s)} \hat{x}_{+}^{(c, \ell)}$ is the predicted measurement, and $\alpha^{(c, \ell, s)}=p_{D}^{(c, \ell, s)}\left(\kappa\left(z_{\theta^{(s)}(\ell)}\right)\right)^{-1}$. Further, the likelihood $\eta_{Z}^{(c, \theta)}(\ell)$ of assigning track label $\ell$ to measurements $\theta(\ell)$ in (12a) is calculated by

$$
\begin{aligned}
& \eta_{Z}^{(c, \theta)}(\ell)=\left\langle p_{+}^{(c)}(\cdot, \ell), \psi_{Z}(\cdot, \ell ; \theta)\right\rangle \\
& =\int \mathcal{N}\left(x ; \hat{x}^{(c, \ell, \theta)}, \underline{P}^{(c, \ell, \theta)}\right) d x \prod_{s=1}^{V}\left(\delta_{0}\left(\theta^{(s)}(\ell)\right) q_{D}^{(s)}\right. \\
& \left.\quad+\left(1-\delta_{0}\left(\theta^{(s)}(\ell)\right)\right) \alpha^{(c, \ell, s)} \mathcal{N}\left(z_{\theta^{(s)}(\ell)} ; z_{+}^{(c, \ell, s)}, \underline{S}^{(c, \ell, s)}\right)\right) \\
& =\prod_{s=1}^{V} \eta_{Z}^{(c, \theta, s)}(\ell) .
\end{aligned}
$$

Since all sensor updated densities $\boldsymbol{\pi}^{(s)}\left(\boldsymbol{X} \mid Z^{(s)}\right)$ in (15b) base on the same predicted density $\boldsymbol{\pi}_{+}(\boldsymbol{X})$, the spaces $\mathbb{C}^{(s)}, \forall s \in\{1 \ldots V\}$ are equal. Expanding (18b) yields

$$
\begin{aligned}
& \tilde{w}_{Z}{ }^{(c, \theta)}(L) \\
& =\frac{\tilde{C}_{w} \prod_{s=1}^{V} C_{d}^{(s)} \delta_{\theta^{(s)-1}\left(\left\{0:\left|Z^{(s)}\right|\right\}\right)}(L) w_{+}^{(c)}(L)\left[\eta_{Z}^{(c, \theta, s)}\right]^{L}}{\prod_{s=1}^{V-1} w_{+}^{(c)}(L)}
\end{aligned}
$$




$$
\begin{aligned}
& =\tilde{C}_{w} C_{w} \delta_{\theta^{-1}(\{0:|Z|\})}(L) w_{+}^{(c)}(L)\left[\eta_{Z}^{(c, \theta)}\right]^{L} \\
& =w_{Z}^{(c, \theta)}(L) \cdot \tilde{C}_{w} C_{w} C_{d}^{-1}=w_{Z}^{(c, \theta)}(L),
\end{aligned}
$$

where $C_{d}^{(s)}$ represents the normalization constants of the single sensor updated densities and $C_{w}=\prod_{s=1}^{V} C_{d}^{(s)}$ is their product. As a result, equality of the weights holds for $\tilde{C}_{w}=C_{d} C_{w}^{-1}$.

Second, we show the equality of the spatial distributions. Insertion of the Gaussian densities into (18c) yields (16a), which has already been traced back to the IMF in Section III-B. Thus, (18d) follows, which equals (13) and completes the proof.

Remark: For similarity reasons, the sensors' detection probability parameters were chosen state-independent. Then, all sensors have to have the same FOV. An extension to consider different FOVs, however, is possible using state-dependent detection probability functions. This extension depends on the specific choice of these functions and the result of the integral in the track label to measurement association likelihoods in (12b). Furthermore, these functions must be known by the centralized fusion center, which has to compensate their effect, e.g., by a constant, if the state-dependent detection probability function from the evaluation section of [9] is chosen for the sensors.

\section{Distributed Implementation of the $\delta$-Generalized Labeled Multi-Bernoulli Filter}

All results derived for GLMB densities are easily applicable using $\delta$-GLMB densities as well, which yields the PM- $\delta$-GLMB filter.

Proposition 3.2: Let us inherit the assumptions of Section III-C, except that all involved distributions are of $\delta$-GLMB type. Then, the updated posterior density $\boldsymbol{\pi}(\boldsymbol{X} \mid Z)$ is a $\delta$-GLMB multi-object distribution and yields

$$
\begin{aligned}
& \boldsymbol{\pi}(\boldsymbol{X} \mid Z)=\Delta(\boldsymbol{X}) \sum_{(I, \xi) \in \mathcal{F}(\mathbb{L}) \times \Xi} \sum_{\theta \in \Theta} \breve{w}_{Z}^{(I, \xi, \theta)} \\
& \cdot \delta_{I}(\mathcal{L}(\boldsymbol{X}))\left[\breve{p}^{(\xi, \theta)}(\cdot \mid Z)\right]^{\boldsymbol{X}},
\end{aligned}
$$

where

$$
\begin{aligned}
\breve{w}_{Z}^{(I, \xi, \theta)} & =\frac{\left.\tilde{C}_{w} \prod_{s=1}^{V} w_{Z^{(s)}}^{\left(I, \xi, \theta^{(s)}\right.}\right)}{\left[w^{(I, \xi)}\right]^{V-1}}, \\
\breve{p}^{(\xi, \theta)}(x, \ell \mid Z) & =\frac{\tilde{C}_{p}(x, \ell \mid Z) \prod_{s=1}^{V} p^{\left(\xi, \theta^{(s)}\right.}\left(x, \ell \mid Z^{(s)}\right)}{\left[p^{(\xi)}(x, \ell)\right]^{V-1}} \\
& =\mathcal{N}\left(x ; \hat{x}^{(\xi, \ell, \theta)}, \underline{P}^{(\xi, \ell, \theta)}\right) .
\end{aligned}
$$

The spatial distribution is fully characterized by its covariance matrix and mean vector, which are defined similar to (18e) and (18f), replacing $c$ by $\xi$.

The proof follows from the proof of Proposition 3.1 by showing equality of (22a) and (14). It is straightforward and, thus, omitted here.

\section{Computationally EfFicient Implementation of THE PM- $\delta$-GLMB FILTER}

In the previous section, we have shown that the mathematical formulation of the GLMB filter update equals that of the proposed PM-GLMB filter update. Note that this is the first closed-form Bayes optimal multi-sensor multi-object filter formulation that allows for a parallelization of the local updates, at the only cost of an additional fusion step. However, due to the exponential growth of the number of hypotheses with time [17], an implementation of these filters in their pure form is infeasible, even though it is possible in theory (cf. [10]). This section details how a computationally efficient implementation can be performed based on the PM- $\delta$-GLMB filter. To do so, first, the system configuration of the distributed implementation of the centralized multi-object estimator with central fusion is defined. Second, pruning and truncation strategies are discussed. Then, the description of our proposed centralized fusion strategy of the distributed sensor updates follows, which features a good trade-off between performance and complexity. The fusion takes advantage of the proposed $k B e s t$ Selections algorithm, and is proven to be optimal. Finally, the computational complexity of the PM- $\delta$-GLMB filter is discussed and compared to state-of-the-art approaches.

\section{A. System Overview}

The PM- $\delta$-GLMB filter is designed for multi-sensor multiobject configurations with centralized fusion and distributed sensors with bidirectional communication links, as can be seen in Fig. 1. According to [1], we denote this configuration the distributed implementation of the centralized multi-object estimator with central fusion. Here, the centralized fusion center holds a global prior density and has knowledge on the trigger time points of the distributed sensors. Based on the global prior, the density is predicted to the next time point including birth handling. This common predicted density is then fed to the sensors, which calculate a local updated posterior density based on their respective sensor measurement. The local posteriors are independent except a common information, which was shown to be the common prediction density in Section III-A. These posteriors are returned to the central fusion node, which calculates the global updated density using (22a). This closes the cycle and another iteration is started.

Within this system, all transmission links use a common interface where only multi-object densities, i.e. weights and spatial distributions, are transmitted. In such a system, if raw data cannot be transmitted and if precision requirements exclude the use of feature-level approaches, the proposed PM- $\delta$-GLMB filter is, to the best of the authors knowledge, the only method that can be applied besides sub-optimal track-totrack fusion strategies like GCI or AMD. Additionally, due to its effectiveness shown in the following sections, this scheme suits also well in completely centralized systems, where it allows for full parallelization and effective truncation.

The birth process is executed globally during the prediction step of the filter. So, by default, a static birth model is assumed. However, if adaptive birth procedures are needed, our scheme can easily be extended. Then, the sensors need to calculate 


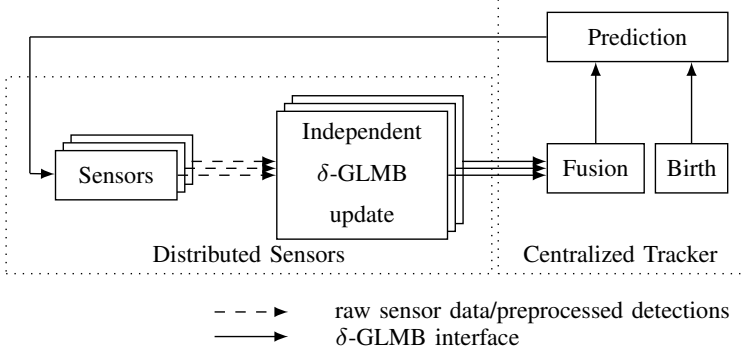

Fig. 1. System overview of a configuration with distributed sensors and centralized tracker. Sensors calculate a local posterior based on the common prediction and local sensor measurements, which is transmitted via a $\delta$-GLMB interface to the central fusion unit and is fused there.

and transmit birth candidate distributions additionally, which would slightly alter the interfaces.

\section{B. Truncation and Pruning Strategies}

The performance of a fusion algorithm is assessable in terms of the computational complexity and the precision of the result. Both are interlinked, since truncation and pruning reduce the precision, but optimize complexity and vice versa. However, the dependencies are complex, since there are multiple ways of tuning complexity and precision, where the truncation and pruning strategy plays a key role. Like in [47], we understand pruning to mean the removal of the most insignificant hypotheses subsequent to their calculation, whereas, like in [9], truncation represents a strategy that simplifies a density prior to its full calculation. Truncation and pruning are commonly used together. Then, naturally, the number of retaining hypotheses after the truncation must be larger than that after the pruning.

The calculation of all hypotheses of an updated density with subsequent pruning is simple and shows good performance, but is still infeasible due to the combinatorial increase of hypotheses. From the aspect of computational complexity, it is better to thin out the hypotheses prior to their calculation, which requires a feasible solution of the ranked assignment problem. An optimal solution to this is given by the Murty algorithm [48], which was proposed to be used for an efficient implementation of the $\delta$-GLMB filter in [10]. However, it remains an NP-hard problem for more than two dimensions and, thus, for multi-sensor scenarios [17]. Therefore, its application to real-world multi-sensor problems is also infeasible. Hence, sub-optimal schemes are required, where the Gibbs sampler has evolved into the solution of choice [16]. Its application reduces the computational complexity in large scenarios significantly. But, as is shown in [17], the straightforward extension of the Gibbs algorithm to the multi-sensor case still yields a computationally infeasible solution. However, the authors present a feasible alternative with parallel update for all synchronous sensor measurements, the SO-Gibbs- $\delta$-GLMB filter [17]. The algorithm samples from marginal distributions, which are approximated to achieve very low computational complexity, whereas precision decreases only slightly.

Truncation acts on a per-hypotheses level, i.e., unfortunately, it can only find the $k$ best assignments based on a certain hypothesis. In consequence, this requires a heuristic to predefine the number of assignments to be calculated per hypothesis. Popular examples preselect these numbers based on the weights of the predicted density [9], based on a PHD look-ahead strategy [10], or using a Gibbs sampler based joint prediction and update strategy [16].

Besides the general choice of methods for truncation and pruning, it is possible to vary the absolute number of truncation and pruning steps as well as the time of execution, which highly depends on the system architecture. Within a centralized IC implementation, the posterior density is truncated and pruned after each sensor update step, which is computationally simple. But it results in the critical limitation that hypotheses can vanish because they are supposed to be insignificant, although later measurements would confirm their significance [17]. The SO-Gibbs- $\delta$-GLMB filter tackles this by finding the approximate optimum of the multi-dimensional assignment problem. Thus, the number of truncation and pruning steps is reduced to one execution per time update. This has been shown in [17] to be superior to the IC- $\delta$-GLMB filter regarding the performance in simulations, whereas the computational complexity remained similar.

Our approach tends towards a similar direction. The local posterior densities are truncated to control the complexity of the local updates. However, no pruning takes place afterwards, since the complexity of the fusion step depends only rarely on the number of hypotheses from the local posterior densities, as is discussed in Section IV-F. So, the multi-object states are described in more detail by the local densities. This results in a fusion that can make better decisions on the retaining hypotheses, since it has more information on hand. Finally, a consecutive sequence of adverse measurements cannot lead to a vanishing hypothesis, because all local density updates are independent of the other posteriors. This means that such situation can only occur if a majority of the sensor measurements misses certain tracks conjointly, which is less likely than a consecutive sequence of a few measurements with the same misdetections. As a result, the probability of a vanishing, but significant hypothesis in our method is reduced significantly in contrast to the IC- $\delta$-GLMB filter.

\section{Fusion of Local Posterior $\delta$-GLMB Densities}

For the centralized fusion of $V$ local posterior updated densities, which are all based on a common prediction, we propose the method summarized in Algorithm 1 and explained in the following. It will later be shown to be computationally efficient, which means it yields a polynomial time complexity.

Fusion Inputs and Output: In consequence of our proposed fusion rule (22), all hypotheses of the fused density $\boldsymbol{\pi}(\boldsymbol{X} \mid Z)$, which is the output of the algorithm, are combinations of hypotheses with common support $(I, \xi)$. Each sensor contributes exactly one hypothesis to each hypothesis in this density. Thus, all local posterior densities from the sensors

$$
\begin{aligned}
\pi^{(s)}\left(\boldsymbol{X} \mid Z^{(s)}\right)=\Delta & \left.(\boldsymbol{X}) \sum_{\theta^{(s)} \in \Theta^{(s)}} w_{Z}^{\left(I, \xi, \theta^{(s)}\right.}\right) \\
& \left.\cdot \delta_{I}(\mathcal{L}(\boldsymbol{X}))\left[p^{\left(\xi, \theta^{(s)}\right.}\right)(\cdot \mid Z)\right]^{\boldsymbol{X}} .
\end{aligned}
$$




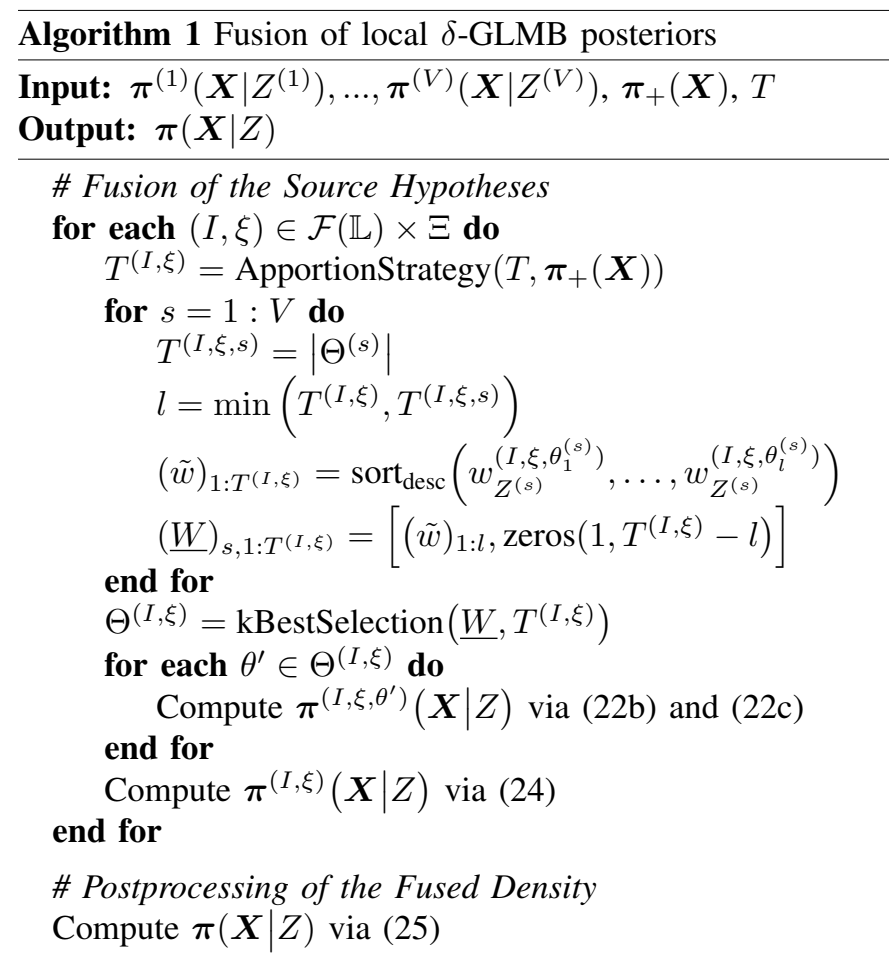

are required as inputs. These hypotheses originate from the predicted hypothesis with the same support, which we denote source hypothesis. A number of $H=|\mathcal{F}(\mathbb{L}) \times \Xi|$ source hypotheses are present in a predicted density, why the predicted density $\boldsymbol{\pi}_{+}(\boldsymbol{X})$ is required as input. Finally, the number of retaining hypotheses $T$ to truncate the density is needed.

Fusion of the Source Hypotheses: First, a strategy to apportion the number of retaining hypotheses $T$ among the $H$ source hypotheses is required. For simplicity reasons, we use the strategy of [10], i.e. $T^{(I, \xi)}=T w^{(I, \xi)}$, when calling $A p$ portionStrategy. Then, the weights of all hypotheses of the $V$ local posterior densities are aligned in a row-wise descending sorted weight matrix $\underline{W} \in \mathbb{R}^{V \times T^{(I, \xi)}}$, as depicted in Fig. 2 . Here, $T^{(I, \xi, s)}$ denotes the number of hypotheses with support $(I, \xi)$ in the $s$-th sensors posterior density. This number may be different from sensor to sensor, e.g. due to gating of measurements. Respective rows with length $l<T^{(i, \xi)}$ are zero-padded by concatenation, denoted by the [.] operator.

Then, using the kBestSelection algorithm proposed in Section IV-D, the subspace $\Theta^{(I, \xi)}$ of $\Theta$ is calculated, which exactly represents the $T^{(I, \xi)}$ best track label to (multi-sensor) associations on support $(I, \xi)$. Each association $\theta^{\prime}$ corresponds to a hypothesis $\boldsymbol{\pi}^{\left(I, \xi, \theta^{\prime}\right)}(\boldsymbol{X} \mid Z)$ in the final density. Its weight and spatial density are calculated by (22b) and (22c), respectively. Finally, these hypotheses are stacked into the partial density on support $(I, \xi)$

$$
\begin{aligned}
\boldsymbol{\pi}^{(I, \xi)}(\boldsymbol{X} \mid Z)= & \sum_{\theta^{\prime} \in \Theta^{(I, \xi)}} \boldsymbol{\pi}^{\left(I, \xi, \theta^{\prime}\right)}(\boldsymbol{X} \mid Z) \\
= & \Delta(\boldsymbol{X}) \sum_{\theta^{\prime} \in \Theta^{(I, \xi)}} w_{Z}^{\left(I, \xi, \theta^{\prime}\right)} \\
& \cdot \delta_{I}(\mathcal{L}(\boldsymbol{X}))\left[p^{\left(\xi, \theta^{\prime}\right)}(\cdot \mid Z)\right]^{\boldsymbol{X}},
\end{aligned}
$$

by summation over the best associations $\Theta^{(I, \xi)}$.

Postprocessing of the Fused Density: The fully fused posterior density $\boldsymbol{\pi}(\boldsymbol{X} \mid Z)$ is simply calculated by summing over all fused partial densities:

$$
\boldsymbol{\pi}(\boldsymbol{X} \mid Z)=\Delta(\boldsymbol{X}) \sum_{(I, \xi) \in \mathcal{F}(\mathbb{L}) \times \Xi} \boldsymbol{\pi}^{(I, \xi)}(\boldsymbol{X} \mid Z) .
$$

Subsequently, the fused posterior density may be sorted and pruned. In either case, normalization has to be done to comply with (22b).

\section{Ranked k-shortest Path Algorithm (kBestSelection)}

We propose the kBestSelection algorithm that is able to efficiently calculate the $k$ best selections in a row-wise sorted weight matrix $\underline{W}$ with positive weights $(\underline{W})_{s, j}$. A valid solution $n$ consists of a selection of one element per row, where the solution's weight is defined by the product of the selected weights and is denoted product weight $w_{n}=\prod_{s=1}^{V}(\underline{W})_{s, j}$. This problem is denoted $k$-shortest path problem and can be solved in polynomial time in general, e.g. using Yen's algorithm [49], [50]. Regarding the $\delta$-GLMB filter, similar problems arise in the prediction step [10] or if the update is approximated [47]. In contrast to these problems, in our case, the underlying graph is an acyclic, directed, vertex-weighted graph of $V-1$ bipartite, complete, and sorted sub-graphs. Thus, it is highly structured. Further, we can assume that in practical scenarios, where computational complexity is an issue, the number of sensors is far below the number of hypotheses, hence $V<<T^{(I, \xi)}$. Then, our algorithm constitutes an approach that highly benefits from these properties and yields an optimal solution with polynomial complexity, as will be discussed in the following two subsections, respectively.

The algorithm is a tree-based method. Each node $N_{n}$ of the tree with the unique index $n$ has a product weight $w_{n}$ and belongs to level $l_{n}$. It represents a unique selection of one matrix element per row, i.e. a potential solution to the $k$ shortest path problem, decoded by the index vector $v_{n}$. That is, the $s$-th vector entry $\left(v_{n}\right)_{s}=y$ selects weight $(\underline{W})_{s, y}$. Each level of the tree comprises all nodes that differ only in one entry by a maximum distance of 1 from at least one of the nodes in the next lower level. The initial node, which is the only node in level 1 , includes the solution with the highest weights from each row, i.e. $v_{1}=[1, \ldots, 1]^{T}$. Figure 3 shows an example for the first two tree levels.

The tree itself is only an auxiliary means and, for efficiency reasons, only partly built by the kBestSelection algorithm. We use the term unexplored to refer to the part of the tree that has not yet been built. While doing so, the algorithm keeps record of two lists, first the list of selected nodes $\mathcal{L}$, hence solutions, and second a list of potential solutions $\mathcal{P}$. Both lists are sorted, meaning that the product weight $w_{n}$ of the elements is descending. In the case of equal product weight, nodes with lower levels are preferred. The kBestSelection algorithm is shown in Algorithm 2. Its inputs are the row-wise sorted weight matrix $\underline{W}$ and the number $k$ of best elements to select, which in the case of the $\delta$-GLMB fusion of algorithm 1 is set to the number of hypotheses $T^{(I, \xi)}$ to keep. The output 


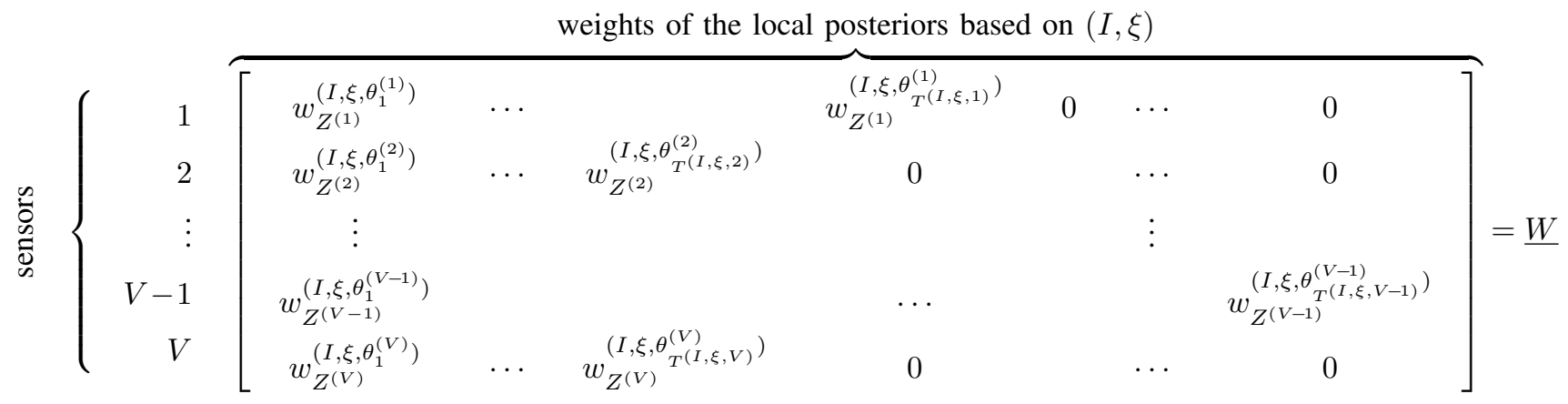

Fig. 2. Exemplary weight matrix $\underline{W}$ for the fusion of local posterior hypotheses from $V$ sensors based on source hypothesis $(I, \xi)$.

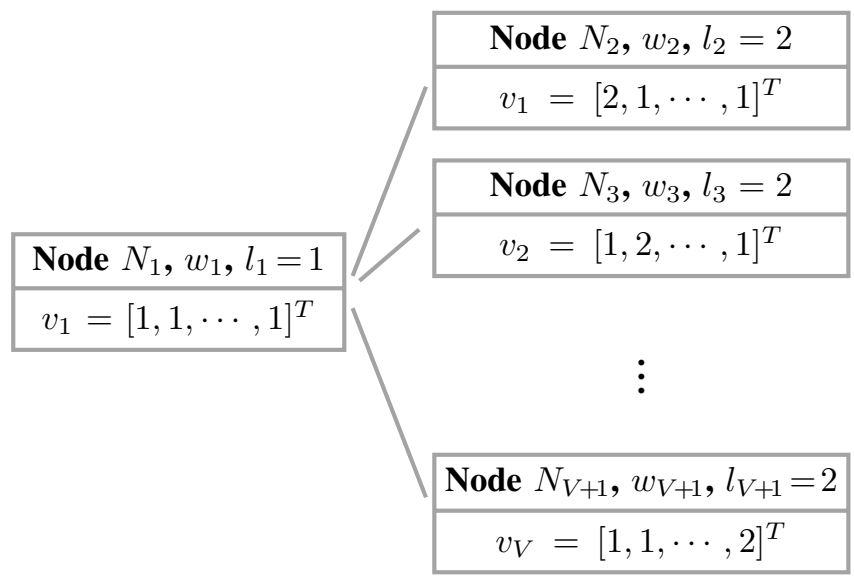

Fig. 3. First two levels of the kBestSelection tree for $V$ sensors. The root node $N_{1}$ represents the elements of the first column of the weight matrix $\underline{W}$.

of the algorithm is the sorted list of selected nodes $\mathcal{L}$, i.e. the subspace $\Theta^{(I, \xi)}$ of the $k$ best hypotheses. The algorithm works like described in the following.

Initialize Tree: The number of sensors is extracted and the sorted weight matrix is cut after $k$ columns, since those elements can never be selected as part of the $k$ best. Then, we initialize the selected nodes list $\mathcal{L}$ and create the root node of the tree with index vector $v_{1}=[1 \ldots 1]^{T}$. It is the trivial first solution and simply selects the highest weighted hypothesis of each sensor, so it is directly sorted into $\mathcal{L}$. Concluding the initialization, the potential candidates list $\mathcal{P}$ is initialized with the best child of the root node.

Find $k-1$ Best Selections: The $k-1$ next best solutions are found by iteratively executing the following strategy $k-1$ times:

i) Take the first, i.e. best, element (pop) from the list of potential solutions $\mathcal{P}$ and append it to the list of selected nodes $\mathcal{L}$.

ii) Find the best child $c$ of the selected node $p$ by following the rules below. A child node $N_{i}$ is one level above its parent $N_{k}$ and must differ from the parent node in exactly one value of the index vector, which is increased by 1 . This means that parents can have $V$ children at most, where the next best child is the one with the largest product weight, which has not yet been added to the tree. However, since equal child nodes can be

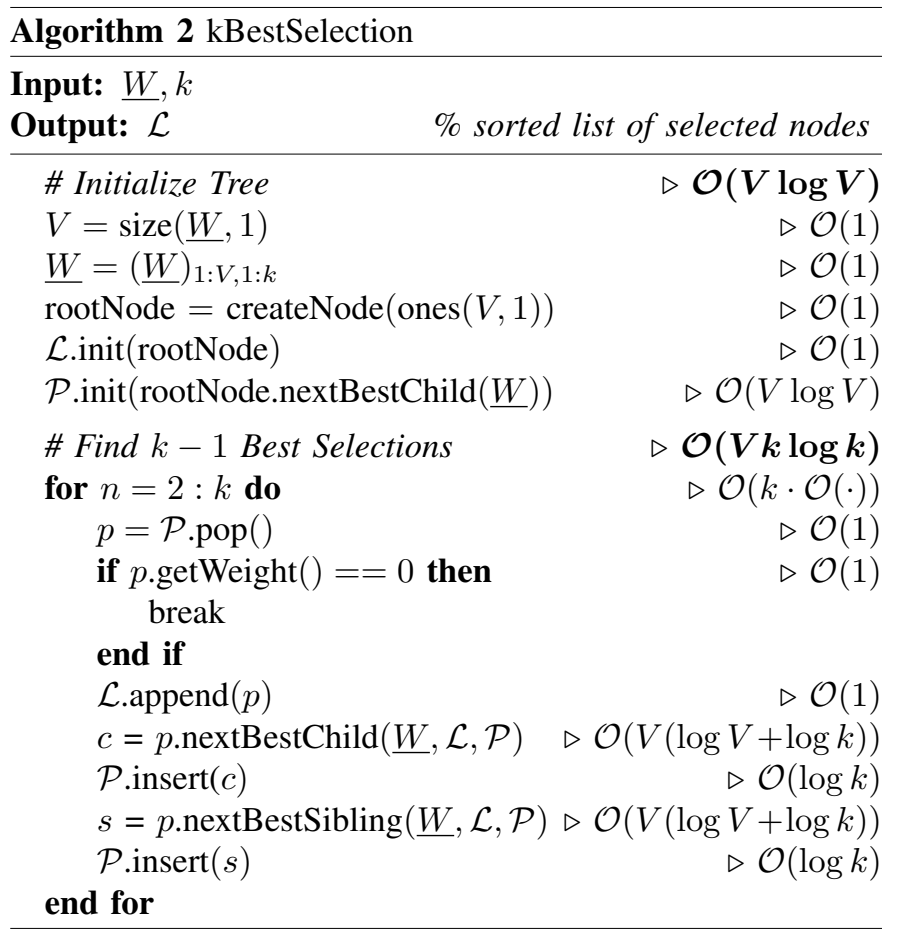

created from different parents, a child node may only be created if it does not already exist in the tree. Thus, if a child is already assigned to another parent, it cannot be reassigned, assigned twice or appear more than once. In such a case, the next best child that satisfies the rules is created and assigned to the parent, as far as it exists. Otherwise, create a dummy node $c$ with zero product weight. Finally, sort the new child $c$ into the list of potential solutions $\mathcal{P}$.

iii) Find the next best sibling $s$ of the selected node $p$ by following the rules below. A sibling is defined as another child of the selected node's parent. To create a sibling node, just call nextBestChild on the parent's node. Finally, sort the sibling node $s$ into the list of potential solutions $\mathcal{P}$ and note that siblings are always on the same level of a tree per definition.

When the algorithm finishes, either $\mathcal{L}$ has length $k$ and, thus, the $k$ best solutions have been found, or the break condition has been fulfilled, i.e. the tree has less than $k$ nodes in total 
and all solutions have been selected.

\section{E. Optimality of the kBestSelection Algorithm}

Proposition 4.1: Given a row-wise sorted weight matrix $\underline{W}$, the proposed algorithm yields the $k$ solutions with highest product weight and, thus, is optimal.

Proof: Proposition 4.1 is proven by mathematical induction, which starts showing that the initial solution is the global best solution and that the first candidate is the global second best solution. In the induction steps, we proof by contradiction that the rules guarantee the global next best solution to be in the list of potential solutions $\mathcal{P}$ and, thus, will always be found.

A) Base Case: Since the initial node selects the largest factor of each row of $\underline{W}$, there cannot be any solution with larger product weight, which is trivial due to the monotonicity of the multiplication. Furthermore, the monotonicity proofs the first candidate node in the sorted potential candidates list to be the second best global solution.

B) Inductive Step: Using the monotonicity of the multiplication like in the base case, it follows that a child has lower or equal product weight than its parent. Further, assume there is a node $N_{u}$ in the unexplored tree that represents the next best global solution, but is not in the list of potential candidates $\mathcal{P}$. Then, our algorithm would fail. However, from the rules of how the tree is created, it follows that such node must either have i) no parent, ii) a parent that has already been selected as a solution, iii) a parent on the potential solutions list $\mathcal{P}$, or iv) a so far unexplored parent. Either of these cases ends in a contradiction, as we show in the following:

i) The presumably best node $N_{u}$ has no parent. Then, it is the root node and, following the initialization rules of the algorithm, must already be a selected solution. Thus, it cannot be unexplored.

ii) The presumably best node's parent is already a selected solution on the solutions list $\mathcal{L}$. Then, $N_{u}$ itself or a sibling of higher product weight must exist on the list of potential solutions $\mathcal{P}$. Thus, $N_{u}$ cannot be unexplored or it is not the next best global solution.

iii) The presumably best node's parent is not a selected solution, but on the potential solutions list $\mathcal{P}$. Then, the parent node has either higher weight than the presumably best node or equal weight, but lower level. Thus, node $N_{u}$ cannot be the next best global solution.

iv) The presumably best node's parent is yet unexplored by the algorithm. Then, node $N_{u}$ cannot be the next best global solution, since its (unexplored) parent node represents a better solution either with lower product weight or with equal product weight, but lower level. Note that you can choose the unexplored parent node as new node $N_{u}$ and repeat this prof of contradiction for this new presumably global best solution.

Thus, such node $N_{u}$ cannot exist, and the node with largest weight in $\mathcal{P}$ always denotes the next best global solution. This proofs the optimality of the proposed algorithm.

\section{F. Discussion of the Computational Complexity}

The complexities for each step of the kBestSelection algorithm as well as the resulting complexities for the initialization and the search process are given in Algorithm 2. Under the assumption that the number of sensors is much smaller than the number of hypotheses, i.e. $V<<k$, which normally is the case for the intended application, the overall complexity is dominated by the for loop and thus yields $\mathcal{O}(V k \log k)$.

For a discussion of the complexity in the multi-object tracking application, we denote the number of measurements by $M$ and $P$ is the number of hypothesized objects. To simplify notation here, $T \triangleq T^{(I, \xi)}$ abbreviates the number of best hypotheses that result from a source hypothesis, i.e. $k=T$. Then, using the PM- $\delta$-GLMB filter, the total computational complexity for each source hypothesis yields $\mathcal{O}\left(V T \log T+T \sum_{s=1}^{V}\left(M^{(s)}+2 P\right)^{4}\right)$, if the Murty algorithm is used, and $\mathcal{O}\left(V T \log T+T P^{2} \sum_{s=1}^{V} M^{(s)}\right)$, if a Gibbs sampler is used. This means that the complexity of the fusion (first summand) is negligible compared to the cost of the sensor updates (second summand) in both cases, resulting in the complexities $\mathcal{O}\left(T \sum_{s=1}^{V}\left(M^{(s)}+2 P\right)^{4}\right)$ (Murty) and $\mathcal{O}\left(T P^{2} \sum_{s=1}^{V} M^{(s)}\right)$ (Gibbs). Note that the $T$ hypotheses of the $V$ sensors have to be sorted prior to the fusion, if not already done by the Murty algorithm or during the pruning. Using a Mergesort algorithm, however, this only adds a complexity of at most $\mathcal{O}(T \log T)$ per sensor [51]. Thus, this has not to be considered further.

These results show that, with respect to complexity, our proposed method can compete with the IC- $\delta$-GLMB filter using a Gibbs sampler, which both have the same complexity. Their complexities increase linearly with the number of sensors and the number of measurements, while they grow quadratic with the number of hypothesized objects. The centralized SO-Gibbs- $\delta$-GLMB filter has slightly lower complexity $\mathcal{O}\left(T P \sum_{s=1}^{V} M^{(s)}\right)$ [17], because it increases only linearly with the number of hypothesized objects. However, the filter comes at the expense of a significantly increased size of the cost and assignment matrices due to the multi-dimensional innovation, which might explain the longer measured execution times compared to the IC- $\delta$-GLMB filter in [17].

\section{Evaluation}

To evaluate the performance of our approach in practice, a simulative study has been carried out, which compares our approach to the IC- $\delta$-GLMB. Since there is no common multisensor benchmark available, two scenarios have been set up: scenario 1, which is based on a scenario from [17], and scenario 2, which was designed to demonstrate the different behavior of the filters in more detail. The basic configuration of both scenarios is similar and visualized in Fig. 4. The observed area is of quadratic shape and has a side length of $800 \mathrm{~m}$. Up to six synchronized sensors are placed around the area, denoted by a black triangle. Within the surveillance region, a maximum of 10 objects are moving around simultaneously following a linear constant velocity path, where Fig. 4 shows the trajectories of the objects of scenario 1 .

The state vector $x_{k}=\left[p_{x}, v_{x}, p_{y}, v_{y}\right]^{T}$ describes the state of an object at time sample $k$, where $p_{x}, p_{y}$ and $v_{x}, v_{y}$ describe the $x$ and $y$ components of the two-dimensional position and velocity, respectively. Each object's motion is modeled by the 


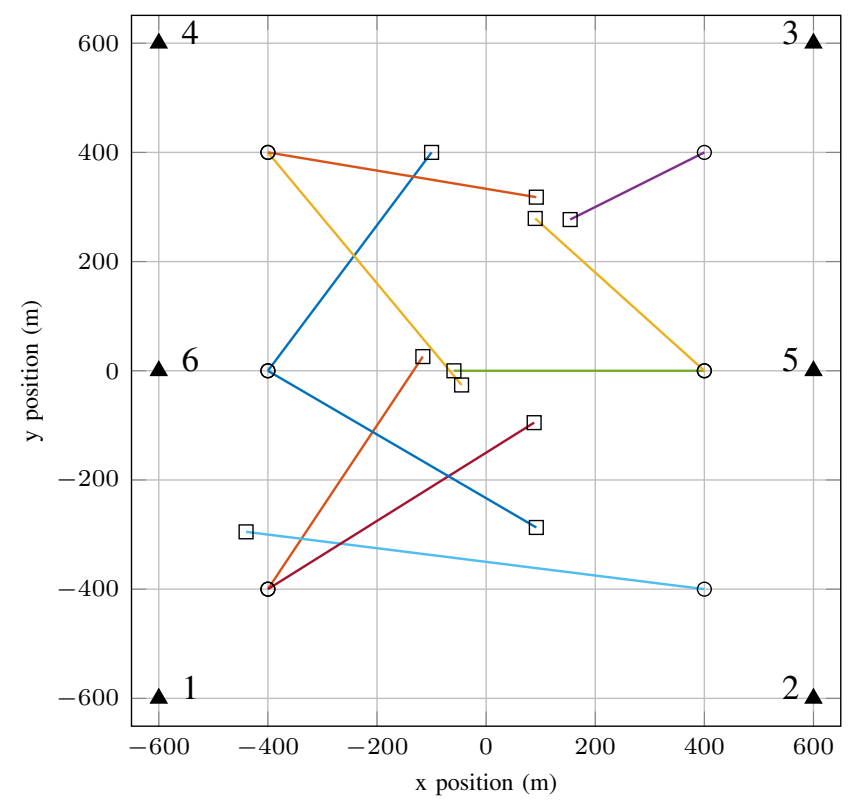

Fig. 4. The surveillance area is shown with all sensor positions and the ground truth paths of all ten objects of scenario 1, where the starting points and end points are marked by circles and rectangles, respectively.

discrete white noise acceleration model [45] using the finite difference state equation $x_{k}=\underline{F} x_{k-1}+\underline{\Gamma} v_{k-1}$ with

$$
\underline{F}=\left[\begin{array}{cccc}
1 & T & 0 & 0 \\
0 & 1 & 0 & 0 \\
0 & 0 & 1 & T \\
0 & 0 & 0 & 1
\end{array}\right], \quad \underline{\Gamma}=\left[\begin{array}{cc}
\frac{T^{2}}{2} & 0 \\
T & 0 \\
0 & \frac{T^{2}}{2} \\
0 & T
\end{array}\right]
$$

The sampling time was chosen to $T=1 \mathrm{~s}$. Within all simulations, the standard deviation of the discrete white noise sequence $v$ was set to $\sigma_{v}=0.2 \mathrm{~m} \mathrm{~s}^{-2}$, and the survival probability was set to $p_{S}=0.98$.

The number of objects varies over time with at most two newly born objects per second. Further, a static birth model is used with six fixed birth regions, where objects can arise. For $i=1, \ldots, 6, p_{B, x}^{(i)}$ and $p_{B, y}^{(i)}$ describe the possible birth positions, which are marked in Fig. 4 by the circles. The birth is modeled by an LMB $\boldsymbol{\pi}_{B}=\left\{r_{B}, p_{B}^{(i)}\right\}_{i=1}^{6}$ with parameters $r_{B}=0.05$ and $p_{B}^{(i)}=\mathcal{N}\left(x ; x_{B}^{(i)}, \underline{Q}_{B}\right)$. Newly born objects are initialized with $x_{B}^{(i)}=\left[p_{B, x}^{(i)}, 0, p_{B, y}^{(i)}, 0\right]^{T}$ and covariance $\underline{Q}_{1}=\operatorname{diag}\left(\left[25 \mathrm{~m}, 5 \mathrm{~m} \mathrm{~s}^{-2}, 25 \mathrm{~m}, 5 \mathrm{~m} \mathrm{~s}^{-2}\right]\right)^{2}$ for scenario 1 and $\underline{Q}_{2}=\operatorname{diag}\left(\left[15 \mathrm{~m}, 5 \mathrm{~m} \mathrm{~s}^{-2}, 15 \mathrm{~m}, 5 \mathrm{~m} \mathrm{~s}^{-2}\right]\right)^{2}$ for scenario 2 , respectively.

All sensors are bearings-only sensors with fixed positions $p_{s}^{(s)}=\left[p_{s, x}^{(s)}, p_{s, y}^{(s)}\right]^{T}$, where the sensor model of Section II-C applies. Thus, independence of the measurements follows and the measurement of an object is distributed by

$$
g_{a}^{(s)}\left(z^{(s)} \mid x, p_{s}^{(s)}\right)=\mathcal{N}\left(z^{(s)}, h_{a}\left(x, p_{s}^{(s)}\right), \underline{R}\right) .
$$

Here,

$$
h_{a}\left(x, p_{s}^{(s)}\right)=\arctan 2\left(\frac{p_{x}-p_{s, x}^{(s)}}{p_{y}-p_{s, y}^{(s)}}\right), \quad \underline{H}_{J}=\left.\frac{\partial h_{a}}{\partial x}\right|_{x},
$$

are the measurement function $h_{a}$ and its Jacobian matrix $\underline{H}_{J}$, respectively. Note that we used the two-dimensional arcus tangent and an Extended Kalman Filter (EKF) to address the non-linearity of the measurement function. The standard deviation of the measurement noise is $\sqrt{\underline{R}}=\sigma_{a}=\pi / 180 \mathrm{rad}$. An object is detected by a chance of $p_{D}=0.67$ and clutter is modeled by a Poisson process with the expected number of false alarms being $\lambda_{c}=7$, which are distributed uniformly according $\mathcal{U}\left(z^{(s)} ;-\pi, \pi\right)$. All simulations are repeated in a Monte Carlo fashion and evaluated using the Optimal Subpattern Assignment metric (OSPA) metric [52] as well as the OSPA $^{(2)}$ metric [53]. Like in [17], the OSPA order is set to $p=1$ and the cut-off is chosen to $c=100 \mathrm{~m}$. Additionally, the OSPA ${ }^{(2)}$ window length is set to $w=20$. Both scenarios are split into two sub-scenarios each, either using only sensors 1 and 2 or all six sensors.

In all scenarios, the Murty algorithm is used in the filter updates. It is configured such that at most $T$ hypotheses retain after the truncated update and approximately $T_{p}$ hypotheses after the pruning. The IC- $\delta$-GLMB applies both, truncation and pruning after each measurement, the PM- $\delta$-GLMB only truncates after the local updates and truncates and prunes after the fusion. That way, we assure equal complexity of the Murty updates, but allow the PM- $\delta$-GLMB to benefit from the more informative update. Additionally, the PM- $\delta$-GLMB filter also benefits from reduced computational cost in general, since all updates are parallelized over the sensors.

\section{A. Scenario 1}

Both sub-scenarios of scenario 1 are repeated 100 times and last $100 \mathrm{~s}$. During each run, 10 objects arise within the first $50 \mathrm{~s}$ with a maximum of two births each $10 \mathrm{~s}$. Beginning from time sample 60 , seven objects vanish with a maximum of two vanishing objects each $10 \mathrm{~s}$. The truncation parameter is set to $T=3000$, from which approximately the best $T_{p}=300$ are kept for the IC- $\delta$-GLMB filter after prediction as well as after each update. The same holds for the PM- $\delta$-GLMB filter, however, the local updated densities are only truncated, pruning solely takes place after the fusion and the prediction. Note that in scenario 1, the improved pruning strategy of [47] has been applied, where additionally the $T_{t o p}=10$ best hypotheses per cardinality are kept as long as their weight is above a threshold of $t_{t h}=1 \mathrm{e}-12$.

The averaged OSPA and OSPA ${ }^{(2)}$ errors of the IC- $\delta$-GLMB filter and the PM- $\delta$-GLMB filter are shown in Fig. 5. As expected, the results with two sensors are worse than those with six sensors. However, two observations are interesting. First, it can clearly be seen that both implementations perform more or less similar in the 2 -sensors case. In contrast, the PM- $\delta$-GLMB filter considerably outperforms the IC- $\delta$-GLMB filter with a higher number of sensors, as can be seen in the 6-sensors case. This confirms the theoretical considerations of Section IV-B, where we argue that in the 2-sensors case, hypotheses vanish equally likely for both filters, whereas, in the 6-sensors case, the PM- $\delta$-GLMB filter looses correct hypotheses less often due to an information overhead. Second, it is, particularly noticeable that both filters' performance regarding the OSPA metric is similar up to time sample $30 \mathrm{~s}$ but not regarding 

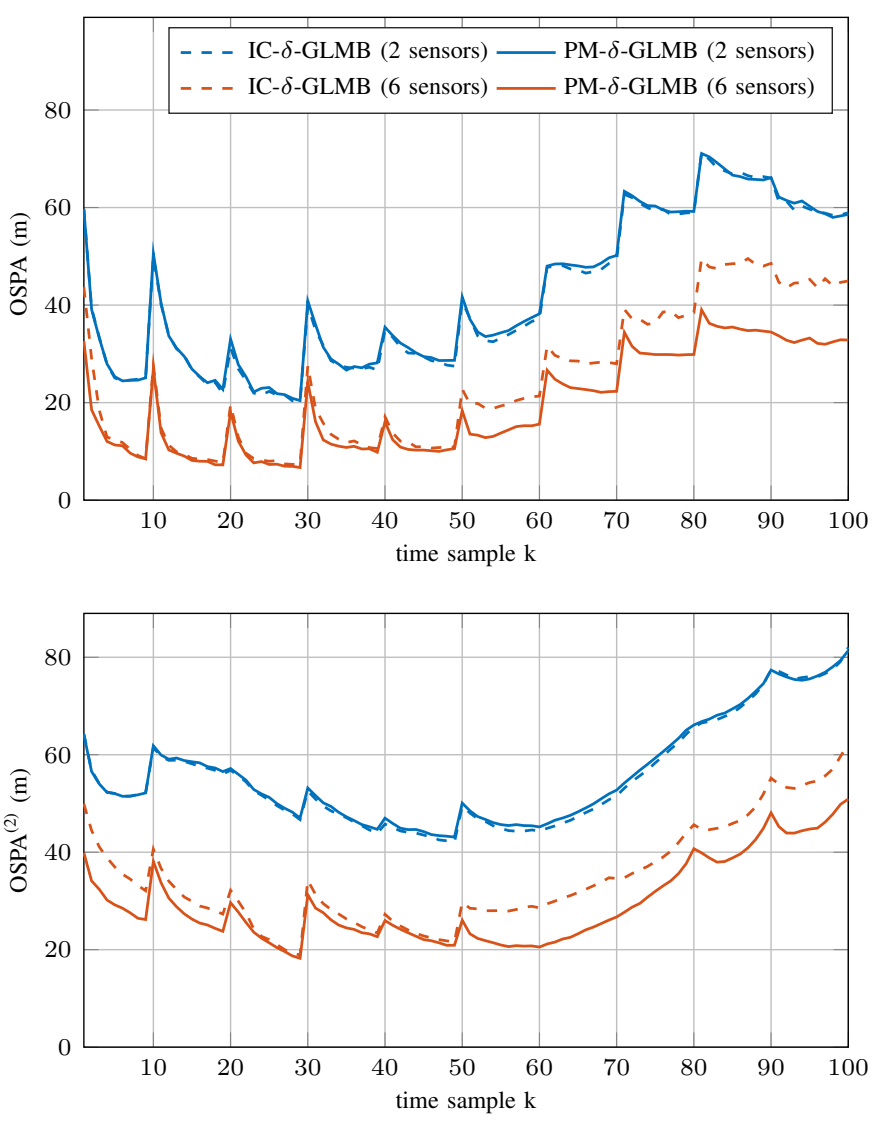

Fig. 5. Averaged OSPA and OSPA(2) error of the IC- $\delta$-GLMB filter and the PM- $\delta$-GLMB filter for scenario 1 .

the $\operatorname{OSPA}^{(2)}$ metric. This reveals that the choice of $T$ and $T_{p}$ is sufficient to represent the true multi-object state in the beginning, and that the PM- $\delta$-GLMB filter is less prone to label switch errors. Later during the simulation, the number of alive objects and, alongside with this, the ambiguity in the track label to measurement assignments increase. Then, vanishing hypotheses in the IC- $\delta$-GLMB filter lead to track depletion, which can be seen in the cardinality estimation in Fig. 6. It shows the true cardinality of scenario 1 in black, as well as the averaged estimated cardinality of the filters in the 6 -sensors case and the $1-\sigma$ bounds of the cardinality estimates as dashed-dotted lines. As can be seen, the IC- $\delta$-GLMB filter tends to underestimate the cardinality and shows a larger uncertainty compared to our proposed PM- $\delta$-GLMB filter.

Summing up, our proposed PM- $\delta$-GLMB filter outperforms the IC- $\delta$-GLMB filter in this scenario. Our filter especially benefits when the sensor number increases, whereas the computational costs increase similarly for both. However, these costs can partly be distributed to the sensor nodes using our PM- $\delta$-GLMB filter. All in all, the PM- $\delta$-GLMB filter benefits from a significantly more informative fusion strategy compared to the IC- $\delta$-GLMB filter, which cannot undo decisions of the prior central measurement update.

\section{B. Scenario 2}

In order to investigate the extinction of significant hypotheses in more detail, a second scenario has been set up. It

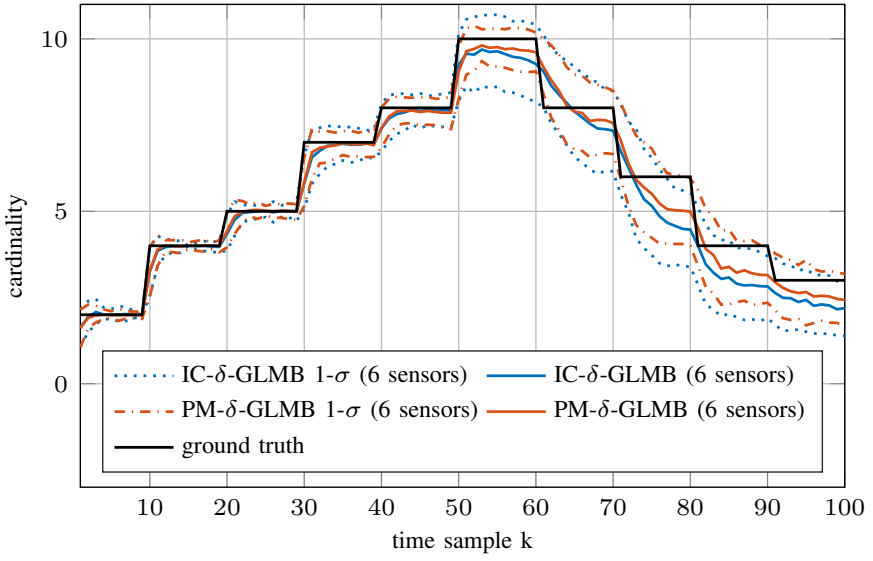

Fig. 6. Cardinality estimation of the IC- $\delta$-GLMB filter and the PM- $\delta$-GLMB filter for the 6-sensors scenario 1 . The true cardinality is shown in black and the $1 \sigma$ bounds using dotted/dash-dotted lines.

lasts $60 \mathrm{~s}$ and is designed such that the probability of track depletion is high. Therefore, the scenario is simplified with two objects only. Both arise at simulation startup and persist until its end in order to eliminate the influence of birth and death on the performance assessment. Moreover, for all objects the initial uncertainty $\underline{Q}_{2}$ is chosen smaller than for scenario 1 . One of the two trajectories equals the cyan one in Fig. 4, the other is created by mirroring the cyan trajectory at an imaginary vertical line with $x$ position 0 . The truncation parameter was set to $T=7$ and pruning to $T_{p}=3$. Thus, in contrast to scenario 1 , the densities cannot describe the situation sufficiently. The results from 250 Monte Carlo (MC) trials are shown in Fig. 7 for both sub-scenarios (2 and 6 sensors, respectively).

Label switching plays a minor role in scenario 2, since there are only two objects that are also clearly separated most of the time. The remaining results from scenario 1 also recur in scenario 2 . The PM- $\delta$-GLMB filter has a significant information advantage that results in a better OSPA and $\mathrm{OSPA}^{(2)}$ metric. As before, this is due to the larger number of combinations the fusion can choose from, since the local posterior densities represent the multi-object state using seven hypotheses, whereas the density of the IC- $\delta$-GLMB filter is pruned to three hypotheses after each measurement update. The fused density is pruned to three hypotheses at the end only. As a result, relevant hypotheses are pruned or truncated by the IC- $\delta$-GLMB filter and, thus, relevant objects die. However, it is very likely that these discarded hypotheses would become significant with one of the next sensor updates, which is inhibited by the severe pruning strategy.

Like in scenario 1, the performance difference between the two filter implementations increases with the number of sensors, making the proposed approach even more advantageous in multi-sensor scenarios with many distributed sensors. Interestingly, the PM- $\delta$-GLMB outperforms the IC- $\delta$-GLMB filter in the 2-sensors case already. Here, the reason is that the tighter specification of the object covariance $\underline{Q}_{2}$ of newly born objects increases the model trust. Both filters benefit from this, since the birth model better reflects the ground truth. But while the IC- $\delta$-GLMB filter compares each measurement on its own 

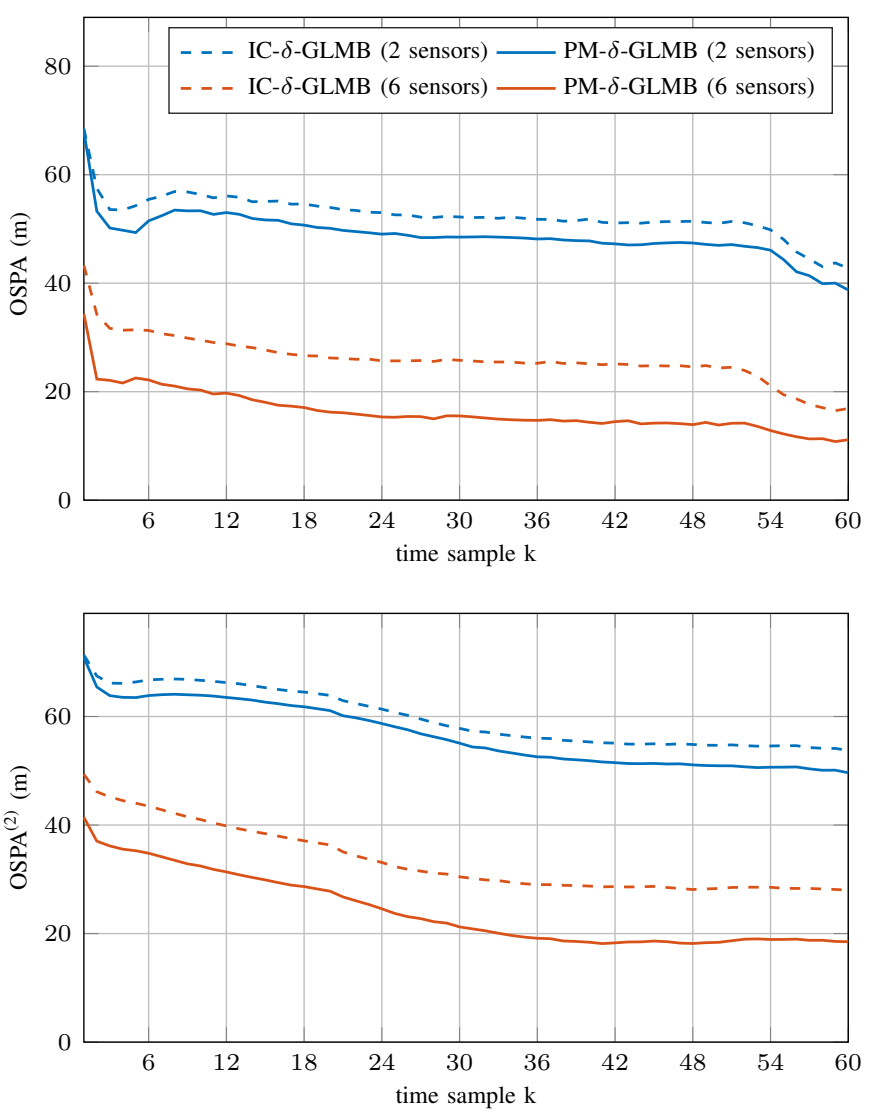

Fig. 7. Averaged OSPA and OSPA ${ }^{(2)}$ error of the IC- $\delta$-GLMB filter and the PM- $\delta$-GLMB filter for scenario 2.

to the model, the PM- $\delta$-GLMB filter compares the model with all measurements altogether, hence, the total model trust is larger and the PM- $\delta$-GLMB filter benefits to a greater extent.

\section{CONCLUSION}

The proposed distributed implementation of the centralized multi-sensor estimator with central fusion (PM- $\delta$-GLMB) is the first multi-sensor multi-object filter using $\delta$-GLMB densities that allows for a distributed and parallel calculation of the sensor updates. Our proposed reformulation of the filter update using the BPCR allows to replace the NP-hard multidimensional $k$-best assignment problem by a much simpler $k$-shortest path problem. We achieved this by the only cost that the sensor truncation acts on a less-informed per-sensor level only. Further, we showed that our approach stands out by its low computational complexity as well as its performance in critical situations, and, thus, outperforms the IC- $\delta$-GLMB filter implementation. The computational complexity of the PM- $\delta$-GLMB filter scales linearly in the number of sensors as well as in the total number of measurements and quadratic in the number of hypothesized objects. Consequently, the proposed method suits perfectly as online multi-sensor multiobject tracker in scenarios with distributed sensors and centralized tracker, like infrastructure based environment modeling for autonomous driving. The proposed algorithm can be seen as the IMF variant of the centralized $\delta$-GLMB filter with the only constraint of synchronized sensors.
In future work, we will evaluate the PM- $\delta$-GLMB filter regarding its performance and complexity in a real world prototype installation with distributed infrastructure sensors for connected autonomous vehicles. This application shall also serve as a basis to compare our method with others, like the IC- $\delta$-GLMB filter or the SO-Gibbs- $\delta$-GLMB filter. Finally, we intend to research how to extend our method to nonsynchronized sensor systems.

\section{REFERENCES}

[1] Y. Bar-Shalom, P. K. Willett, and X. Tian, Tracking and Data Fusion: A Handbook of Algorithms. Storrs, Conn: YBS Publishing, 2011.

[2] R. P. S. Mahler, Statistical Multisource-Multitarget Information Fusion. Norwood, MA, USA: Artech House, Inc., 2007.

[3] S. R. E. Datondji, Y. Dupuis, P. Subirats, and P. Vasseur, "A survey of vision-based traffic monitoring of road intersections," IEEE Trans. Intell. Transp. Syst., vol. 17, no. 10, pp. 2681-2698, 2016.

[4] M. Herrmann, A. Piroli, J. Strohbeck, J. Müller, and M. Buchholz, "LMB filter based tracking allowing for multiple hypotheses in object reference point association," in Proc. IEEE Int. Conf. Multisensor Fusion and Integrat. Intell. Syst., 2020, pp. 197-203.

[5] J. Müller, M. Gabb, and M. Buchholz, "A subjective-logic-based reliability estimation mechanism for cooperative information with application to IV's safety," in Proc. IEEE Intell. Veh. Symp., 2019, pp. 1940-1946.

[6] M. Goldhammer, E. Strigel, D. Meissner, U. Brunsmann, K. Doll, and K. Dietmayer, "Cooperative multi sensor network for traffic safety applications at intersections," in Proc. IEEE Conf. Intell. Transp. Syst., 2012.

[7] M. Buchholz, J. Müller, M. Herrmann, J. Strohbeck, B. Völz, M. Maier, J. Paczia, O. Stein, H. Rehborn, and R.-W. Henn, "Handling occlusions in automated driving using a MEC server-based environment model from infrastructure sensors," IEEE Intell. Transp. Syst. Mag., 2021.

[8] N. Kaempchen, M. Buehler, and K. Dietmayer, "Feature-level fusion for free-form object tracking using laserscanner and video," in Proc. IEEE Intell. Veh. Symp., 2005, pp. 453-458.

[9] B.-T. Vo and B.-N. Vo, "Labeled random finite sets and multi-object conjugate priors," IEEE Trans. Signal Process., vol. 61, no. 13, pp. 3460-3475, 2013.

[10] B.-N. Vo, B.-T. Vo, and D. Phung, "Labeled random finite sets and the bayes multi-target tracking filter," IEEE Trans. Signal Process., vol. 62, no. 24, pp. 6554-6567, 2014.

[11] R. P. S. Mahler, "Multitarget bayes filtering via first-order multitarget moments," IEEE Trans. Aerosp. Electron. Syst., vol. 39, no. 4, pp. 1152 $1178,2003$.

[12] R. P. S. Mahler, "PHD filters of higher order in target number," IEEE Trans. Aerosp. Electron. Syst., vol. 43, no. 4, pp. 1523-1543, 2007.

[13] C. Fantacci and F. Papi, "Scalable multisensor multitarget tracking using the marginalized $\delta$-GLMB density," IEEE Signal Process. Lett., vol. 23, no. 6, pp. 863-867, 2016.

[14] S. Reuter, B. Vo, B. Vo, and K. Dietmayer, "The labeled multi-bernoulli filter," IEEE Trans. Signal Process., vol. 62, no. 12, pp. 3246-3260, 2014.

[15] B.-T. Vo, B.-N. Vo, and A. Cantoni, "The cardinality balanced multitarget multi-bernoulli filter and its implementations," IEEE Trans. Signal Process., vol. 57, no. 2, pp. 409-423, 2009.

[16] B.-N. Vo, B.-T. Vo, and H. G. Hoang, "An efficient implementation of the generalized labeled multi-bernoulli filter," IEEE Trans. Signal Process. vol. 65 , no. 8, pp. 1975-1987, 2017.

[17] B.-N. Vo, B.-T. Vo, and M. Beard, "Multi-sensor multi-object tracking with the generalized labeled multi-bernoulli filter," IEEE Trans. Signal Process., vol. 67, no. 23, pp. 5952-5967, 2019.

[18] B. Wei, B. Nener, W. Liu, and L. Ma, "Centralized multi-sensor multitarget tracking with labeled random finite sets," in Proc. IEEE Int. Conf. Contr., Automat. and Inf. Sci. (ICCAIS), 2016, pp. 82-87.

[19] C. Genest and J. V. Zidek, "Combining probability distributions: A critique and an annotated bibliography," Statistical Sci., vol. 1, no. 1, pp. 114-135, 1986.

[20] T. Bailey, S. Julier, and abriel Agamennoni, "On conservative fusion of information with unknown non-gaussian dependence," in Proc. 15th IEEE Int. Conf. Inf. Fusion, 2012, pp. 1876-1883.

[21] T. Li, X. Wang, Y. Liang, and Q. Pan, "On arithmetic average fusion and its application for distributed multi-bernoulli multitarget tracking," IEEE Trans. Signal Process., vol. 68, pp. 2883-2896, 2020. 
[22] R. P. S. Mahler, "Optimal/robust distributed data fusion: a unified approach," in Proc. SPIE, vol. 4052, 2000.

[23] M. B. Hurley, "An information theoretic justification for covariance intersection and its generalization," in Proc. 5th IEEE Int. Conf. Inf. Fusion, vol. 1, 2002, pp. 505-511.

[24] A. Buonviri, M. York, K. LeGrand, and J. Meub, "Survey of challenges in labeled random finite set distributed multi-sensor multi-object tracking," in Proc. IEEE Aerosp. Conf., 2019, pp. 1-12.

[25] G. Battistelli, L. Chisci, C. Fantacci, A. Farina, and B.-N. Vo, "Average kullback-leibler divergence for random finite sets," in Proc. 18th IEEE Int. Conf. Inf. Fusion, 2015, pp. 1359-1366.

[26] K. Da, T. Li, Y. Zhu, H. Fan, and Q. Fu, "Recent advances in multisensor multitarget tracking using random finite set," Front. Inf. Technol. Electron. Eng., vol. 22, no. 1, pp. 5-24, 2021.

[27] M. Uney, D. E. Clark, and S. J. Julier, "Distributed fusion of PHD filters via exponential mixture densities," IEEE J. Sel. Topics Signal Process., vol. 7, no. 3, pp. 521-531, 2013.

[28] G. Battistelli, L. Chisci, C. Fantacci, A. Farina, and A. Graziano, "Consensus CPHD Filter for Distributed Multitarget Tracking," IEEE J. Sel. Topics Signal Process., vol. 7, no. 3, pp. 508-520, 62013.

[29] B. Wang, W. Yi, R. Hoseinnezhad, S. Li, L. Kong, and X. Yang, "Distributed fusion with multi-bernoulli filter based on generalized covariance intersection," IEEE Trans. Signal Process., vol. 65, no. 1, pp. 242-255, 2017.

[30] W. Yi, S. Li, B. Wang, R. Hoseinnezhad, and L. Kong, "Computationally efficient distributed multi-sensor fusion with multi-bernoulli filter," IEEE Trans. Signal Process., vol. 68, pp. 241-256, 2020.

[31] S. Li, W. Yi, R. Hoseinnezhad, G. Battistelli, B. Wang, and L. Kong, "Robust Distributed Fusion With Labeled Random Finite Sets," IEEE Trans. Signal Process., vol. 66, no. 2, pp. 278-293, 2018.

[32] C. Fantacci, B.-N. Vo, B.-T. Vo, G. Battistelli, and L. Chisci, "Robust fusion for multisensor multiobject tracking," IEEE Signal Process. Lett., vol. 25, no. 5, pp. 640-644, 2018.

[33] S. Li, G. Battistelli, L. Chisci, W. Yi, B. Wang, and L. Kong, "Computationally efficient multi-agent multi-object tracking with labeled random finite sets," IEEE Trans. Signal Process., vol. 67, no. 1, pp. 260-275, 2019.

[34] T. Kropfreiter and F. Hlawatsch, "A probabilistic label association algorithm for distributed labeled multi-bernoulli filtering," in Proc. $23 \mathrm{rd}$ IEEE Int. Conf. Inf. Fusion, 2020.

[35] W. Yi, G. Li, and G. Battistelli, "Distributed multi-sensor fusion of PHD filters with different sensor fields of view," IEEE Trans. Signal Process., vol. 68, pp. 5204-5218, 2020.

[36] J. Y. Yu, M. Coates, and M. Rabbat, "Distributed multi-sensor CPHD filter using pairwise gossiping," in Proc. IEEE Int. Conf. Acoust., Speech, Signal Process. (ICASSP), 2016.

[37] L. Gao, G. Battistelli, and L. Chisci, "Multiobject fusion with minimum information loss," IEEE Signal Process. Lett., vol. 27, pp. 201-205, 2020.

[38] - "Fusion of labeled RFS densities with minimum information loss," IEEE Trans. Signal Process., vol. 68, pp. 5855-5868, 2020.

[39] A. Scheel, S. Reuter, and K. Dietmayer, "Vehicle tracking using extended object methods: An approach for fusing radar and laser," in Proc. IEEE Int. Conf. on Robot. and Automat., 2017, pp. 231-238.

[40] M. Herrmann, J. Müller, J. Strohbeck, and M. Buchholz, "Environment modeling based on generic infrastructure sensor interfaces using a centralized labeled-multi-bernoulli filter," in Proc. IEEE Conf. Intell. Transp. Syst., 2019, pp. 2414-2420.

[41] R. Kruse, E. Schwecke, and J. Heinsohn, Uncertainty and Vagueness in Knowledge Based Systems. Springer Berlin Heidelberg, 1991.

[42] R. P. S. Mahler, "Approximate multisensor CPHD and PHD filters," in Proc. 13th IEEE Int. Conf. Inf. Fusion, 2010, pp. 1-8.

[43] - Advances in Statistical Multisource-Multitarget Information Fusion. Norwood, MA, USA: Artech House Publishers, 2014.

[44] M. E. Liggins, Chee-Yee Chong, I. Kadar, M. G. Alford, V. Vannicola and S. Thomopoulos, "Distributed fusion architectures and algorithms for target tracking," Proceedings of the IEEE, vol. 85, no. 1, pp. 95-107, 1997.

[45] Y. Shalom, X.-R. Li, and T. Kirubarajan, Estimation with Applications to Tracking and Navigation. New York: Wiley, 2001.

[46] Y. C. Ho and R. C. K. Lee, "A Bayesian Approach to Problems in Stochastic Estimation and Control," IEEE Trans. Autom. Control, vol. 9, no. 4, pp. 333-339, 1964

[47] M. Beard, B. Vo, and B. Vo, "Bayesian multi-target tracking with merged measurements using labelled random finite sets," IEEE Signal Process. Lett., vol. 63, no. 6, pp. 1433-1447, 32015.
[48] K. Murty, "An algorithm for ranking all the assignments in order of increasing cost," Operations Research, vol. 16, no. 3, pp. 682-687, 1968.

[49] J. Y. Yen, "Finding the k shortest loopless paths in a network," Manage. Sci., vol. 17, no. 11, pp. 712-716, 1971.

[50] D. Eppstein, "Finding the k shortest paths," in Proc. 35th Annu. Symp. Found. Comput. Sci. IEEE Comput. Soc. Press, 1994.

[51] J. Katajainen and J. L. Träff, "A meticulous analysis of mergesort programs," in Proc. 2nd Int. Conf. Algorithms and Complexity (CIAC). Springer Berlin Heidelberg, 1997, pp. 217-228.

[52] D. Schuhmacher, B. Vo, and B. Vo, "A consistent metric for performance evaluation of multi-object filters," IEEE Trans. Signal Process., vol. 56, no. 8, pp. 3447-3457, 2008.

[53] M. Beard, B. T. Vo, and B.-N. Vo, "OSPA(2): Using the OSPA metric to evaluate multi-target tracking performance," in Proc. IEEE Int. Conf. Contr., Automat. and Inf. Sci. (ICCAIS), 2017.

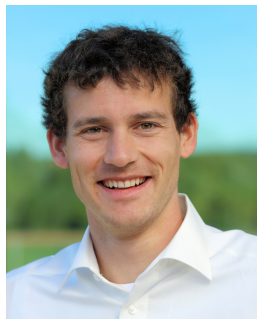

Martin Herrmann received the B.Sc. degree in Media Technology in 2014 from Ilmenau University of Technology and his M.Sc. degree in Electrical Engineering in 2017 from Ulm University, both located in Germany. Since 2017 he is a research assistant at the Institute of Measurement, Control and Microtechnology at Ulm University in Germany. His research interests are sensor data fusion, filtering and estimation, signal processing and environment perception for autonomous driving.

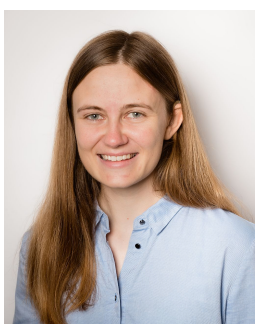

Charlotte Hermann received the B.Sc. degree in 2017 and the M.Sc degree in 2019 in electrical engineering from Ulm University, Germany. Afterwards, she joined the Institute of Measurement, Control and Microtechnology at Ulm University as a research assistant. Her main research topics are multi-objecttracking and sensor data fusion.

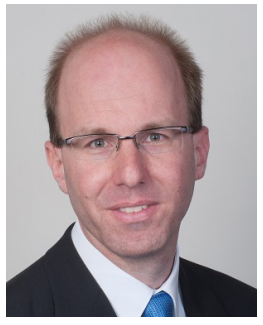

Michael Buchholz received his Diploma degree in Electrical Engineering and Information Technology as well as his Ph.D. from the faculty of Electrical Engineering and Information Technology at Karlsruhe Institute of Technology, Germany. Since 2009 , he is serving as a research group leader and lecturer at the Institute of Measurement, Control and Microtechnology at Ulm University, Germany. His research interests comprise connected automated driving, electric mobility, modeling and control of mechatronic systems, and system identification. 